\title{
LIE ALGEBRA DEFORMATIONS IN CHARACTERISTIC 2
}

\author{
SOFIANE BOUARROUDJ ${ }^{1}$, ALEXEI LEBEDEV ${ }^{2}$, DIMITRY LEITES $^{3}$, IRINA SHCHEPOCHKINA ${ }^{4}$
}

\begin{abstract}
Of four types of Kaplansky algebras, type- 2 and type- 4 algebras have previously unobserved $\mathbb{Z} / 2$-gradings: nonlinear in roots. A method assigning a simple Lie superalgebra to every $\mathbb{Z} / 2$-graded simple Lie algebra in characteristic 2 is illustrated by seven new series. Type- 2 algebras and one of the two type- 4 algebras are demystified as nontrivial deforms (the results of deformations) of the alternate Hamiltonian algebras. The type-1 Kaplansky algebra is recognized as the derived of the nonalternate version of the Hamiltonian Lie algebra, the one that preserves a tensorial 2-form, not an exterior one.

Deforms corresponding to nontrivial cohomology classes can be isomorphic to the initial algebra, e.g., we confirm Grishkov's implicit claim and explicitly describe the Jurman algebra as such a "semitrivial" deform of the derived of the alternate Hamiltonian Lie algebra. This paper helps to sharpen the formulation of a conjecture describing all simple finite-dimensional Lie algebras over any algebraically closed field of nonzero characteristic and supports a conjecture of Dzhumadildaev and Kostrikin stating that all simple finitedimensional modular Lie algebras are either of "standard" type or deforms thereof.

In characteristic 2 , we give sufficient conditions for the known deformations to be semitrivial.
\end{abstract}

\section{INTRODUCTION}

Hereafter, $\mathbb{K}$ is an algebraically closed field of characteristic $p>0$ unless otherwise stated. The letter $p$ also denotes "momenta" indeterminates but confusion is impossible.

1.1. Preparatory information. Assuming that $p^{\infty}=\infty$ and $\mathbb{N}=\{1,2, \ldots\}$, we designate

$$
\begin{aligned}
& \mathcal{O}(m ; \underline{N}):=\mathbb{K}[u ; \underline{N}]:=\operatorname{Span}_{\mathbb{K}}\left(u^{(r)} \mid r_{i}<p^{N_{i}}\right) \\
& \text { for } u=\left(u_{1}, \ldots, u_{m}\right) \text { and } r=\left(r_{1}, \ldots, r_{m}\right) \text { and any } m \text {-tuple } \underline{N}=\left(N_{1}, \ldots, N_{m}\right),
\end{aligned}
$$

where $N_{i} \in \mathbb{N} \cup \infty$ for any $i$, the addition is natural, and the product is given by

$$
u^{(\underline{r})} \cdot u^{(\underline{s})}=\left(\begin{array}{c}
\underline{r}+\underline{s} \\
\underline{r}
\end{array}\right) u^{(\underline{r}+\underline{s})}, \text { where }\left(\begin{array}{c}
\underline{r}+\underline{s} \\
\underline{r}
\end{array}\right):=\prod_{i=1}^{m}\left(\begin{array}{c}
r_{i}+s_{i} \\
r_{i}
\end{array}\right) .
$$

The elements of the algebra $\mathcal{O}(m ; \underline{N})$ of divided powers serve as "functions" over $\mathbb{K}$. The shearing vector with smallest coordinates

$$
\underline{N}_{s}=(1, \ldots, 1)
$$

is of particular interest (see [Vi1, BLLS]). Only one of the algebras of divided powers $\mathcal{O}(n ; \underline{N})$ is indeed generated by the indeterminates declared: if $\underline{N}=\underline{N}_{s}$. Otherwise, the list of generators consists of $u_{i}^{\left(p^{k_{i}}\right)}$ for all $i$ and $k_{i}$ such that $1 \leq k_{i}<N_{i}$. We define distinguished partial derivatives by setting

$$
\partial_{i}\left(u_{j}^{(k)}\right)=\delta_{i j} u_{j}^{(k-1)} \quad \text { for any } k<p^{N_{j}} .
$$

1991 Mathematics Subject Classification. Primary 17B50, 17B20; Secondary 17B25, 17B55, 17B56.

Key words and phrases. Lie algebra, characteristic 2, Kostrikin-Shafarevich conjecture, Jurman algebra, Kaplansky algebra, deformation. 
Let $\operatorname{vect}(m ; \underline{N}):=\mathfrak{d e r}_{\text {dist }}(\mathcal{O}(m ; \underline{N}))$ be the general vectorial Lie algebra spanned by all distinguished derivations $f_{i} \partial_{i}$, where $f_{i} \in \mathcal{O}(m ; \underline{N})$; let $\mathfrak{s v e c t}(m ; \underline{N})$ be its subalgebra of divergence-free derivations. Various vectorial Lie algebras are complete or partial Cartan prolongs, i.e., the results of generalized prolongation procedures.

1.1.1. Complete Cartan prolongations. For details, see [Shch]. Let $D S^{k}$ be the operation of raising to the $k$ th divided symmetric power and $D S^{\bullet}:=\underset{k \geq 0}{\oplus} D S^{k}$; we set

$$
\begin{aligned}
& i: D S^{k+1}\left(\mathfrak{g}_{-1}\right)^{*} \otimes \mathfrak{g}_{-1} \longrightarrow D S^{k}\left(\mathfrak{g}_{-1}\right)^{*} \otimes \mathfrak{g}_{-1}^{*} \otimes \mathfrak{g}_{-1}, \\
& j: D S^{k}\left(\mathfrak{g}_{-1}\right)^{*} \otimes \mathfrak{g}_{0} \longrightarrow D S^{k}\left(\mathfrak{g}_{-1}\right)^{*} \otimes \mathfrak{g}_{-1}^{*} \otimes \mathfrak{g}_{-1}
\end{aligned}
$$

as the natural maps. Let the $(k, \underline{N})$ th prolong of the pair $\left(\mathfrak{g}_{-1}, \mathfrak{g}_{0}\right)$ be

$$
\mathfrak{g}_{k, \underline{N}}=\left(j\left(D S^{\bullet}\left(\mathfrak{g}_{-1}\right)^{*} \otimes \mathfrak{g}_{0}\right) \cap i\left(D S^{\bullet}\left(\mathfrak{g}_{-1}\right)^{*} \otimes \mathfrak{g}_{-1}\right)\right)_{k, \underline{N}},
$$

where the subscript $k$ in the right-hand side singles out the component of degree $k$. It is easy to show that if the $\mathfrak{g}_{0}$-module $\mathfrak{g}_{-1}$ is faithful, then $\left(\mathfrak{g}_{-1}, \mathfrak{g}_{0}\right)_{*, \underline{N}}=\underset{k}{\oplus} \mathfrak{g}_{k, \underline{N}}$ is a Lie subalgebra in $\mathfrak{v e c t}\left(\operatorname{dim} \mathfrak{g}_{-1} ; \underline{N}\right)$; it is called the (complete) Cartan prolong of the pair $\left(\mathfrak{g}_{-1}, \mathfrak{g}_{0}\right)$. A partial prolong is a subalgebra of $\left(\mathfrak{g}_{-1}, \mathfrak{g}_{0}\right)_{*, \underline{N}}$ generated by $\mathfrak{g}_{-1}, \mathfrak{g}_{0}$, and a $\mathfrak{g}_{0}$-submodule of $\mathfrak{g}_{1}$.

1.1.2. Lie algebras of Hamiltonian series. A detailed description of several types of Hamiltonian series, their divergence-free subalgebras, their central extensions-Poisson algebras, and their simple derived in characteristic 2 can be found in $[\mathrm{LeP}]$. Here, we briefly recall that a given symmetric bilinear form $B$ on the space $V$ is said to be alternate if $B(v, v)=0$ for any $v \in V$ and nonalternate otherwise. The normal shapes of nondegenerate bilinear forms $B$ whose Gram matrices are also denoted by $B$ are denoted by $\Pi(n)$ and $I(n)$ if reduced to the side and main diagonal, respectively, where $n=\operatorname{dim} V$. The orthogonal Lie algebras $\mathfrak{o}_{B}(V)$ that preserve these normal forms are denoted by $\mathfrak{o}_{\Pi}(V)$ and $\mathfrak{o}_{I}(V)$, respectively. If $n$ is odd, then there is only one equivalence class of nondegenerate symmetric bilinear forms, and we can hence drop the subscript $B$ in $\mathfrak{o}_{B}(V)$; if $\operatorname{dim} V$ is even, then there are two equivalence classes of nondegenerate symmetric forms.

The Hamiltonian Lie algebra can be alternate $\mathfrak{h}_{\Pi}(V ; \underline{N})$ or nonalternate $\mathfrak{h}_{I}(V ; \underline{N})$ depending on the type of the differential 2 -form 11 the algebra preserves by means of the Lie derivative. We often write $\mathfrak{h}_{B}(n ; \underline{N})$ instead of $\mathfrak{h}_{B}(V ; \underline{N})$. Both $\mathfrak{h}_{\Pi}(V ; \underline{N})$ and $\mathfrak{h}_{I}(V ; \underline{N})$ have divergence-free subalgebras described, together with a history of earlier partial discoveries, in $[\mathrm{LeP}]$.

In this paper, we describe $\mathfrak{h}_{B}(V ; \underline{N})$ as the Cartan prolong $\left(V, \mathfrak{o}_{B}(V)\right)_{*, \underline{N}}$ with the multiplication given by the Poisson bracket of generating functions

$$
\{F, G\}_{B}=\sum_{1 \leq i, j \leq n} B_{i j} \frac{\partial F}{\partial x_{i}} \frac{\partial G}{\partial x_{j}} \text { for any } F, G \in \mathcal{O}(n ; \underline{N}), \text { where }\left(B_{i j}\right)=B .
$$

The elements of $\mathfrak{h}_{B}(V ; \underline{N})$ can be realized by vector fields

$$
H_{F}=\sum_{1 \leq i, j \leq n} B_{i j} \frac{\partial F}{\partial x_{i}} \frac{\partial}{\partial x_{j}} \text { for any } F \in \mathcal{O}(n ; \underline{N}),
$$

where $\left(B_{i j}\right)_{i, j=1}^{n}=B$. The Lie algebra whose space is $\mathcal{O}(n ; \underline{N})$ with the bracket (6) is called the Poisson algebra if $B \not I$; it is a central extension of $\mathfrak{h}_{B}(n ; \underline{N})$ for $B \sim \Pi$.

We note that although $\mathfrak{h}_{I}(n ; \underline{N})$ is well defined, there is no Lie algebra $\mathfrak{p o}_{I}(n ; \underline{N})$ with the bracket (6). Indeed, the bracket should be antisymmetric, i.e., alternate, while

\footnotetext{
${ }^{1}$ It was shown in $[\mathrm{LeP}$ that this 2 -form is the sum of products of 1 -forms, the product being either exterior (alternate case) or tensor (nonalternate case).
} 
$\left\{x_{i}, x_{i}\right\}_{I}=1$, not 0 . More on possible brackets corresponding to the alternate bilinear form $B$ can be found in LeP and subsec. 3.2.

1.2. Overview of the situation. Even the incomplete stock of nonisomorphic species in the zoo of simple finite-dimensional Lie algebras for $p=2$ was until recently considered uncomfortably numerous (see the introduction in [S]). It has many more exhibits than would have been considered "normal" if the classification in cases $p>3$ were taken as a "norm." A final touch in the proof of the classification can be found in [BGP].

The improved version of the Kostrikin-Shafarevich conjecture due to Dzumadildaev and Kostrikin [KD] states that for any $p>0$,

\section{all simple Lie algebras are either of "standard" type or deforms (the results of deformations) thereof.}

The improved conjecture definitely embraces $p \geq 5$ as proved in [KD], where the Melikyan algebras are identified as deforms of the Poisson algebras. The claim in KuJa identifying the simple Ermolaev algebras as deforms of the contact algebras supports the conjecture (8) for $p=3$. The conjecture (8) seems plausible if, for $p=2$ and 3, we enlarge the stock of examples "standard" for $p \geq 5$ by exhibits from GL, SkT1, BGL1, BGL3, LeP, BGLLS, BGLLS1, mostly found after (8) was formulated. Therefore, we must find out which simple Lie algebras are "standard" from the standpoint of (18), and solve a "small technical problem" of describing all nonisomorphic deforms.

A conjectural description of "standard", hence, all (together with their deforms) simple finite-dimensional Lie algebras over $\mathbb{K}$ for $p=2$, although longer than that for $p>3$, is possible to grasp. This conjecture stemmed from an idea that had already led to the classification of simple Lie superalgebras of polynomial vector fields over $\mathbb{C}$ (see [LSh1]). The new conjecture yielded new examples for $p=3$ (see the arXiv version of [GL]).

For $p=2$, the new conjecture (see [Ltow] for a briefly formulated version) gathers all examples known to us in describable groups and indicates ways to obtain new examples. Apart from these and several new examples given in [BGLLS1, there are also known examples (due to Kaplansky, Shen, Skryabin, Brown, Jurman, Vaughan-Lee, and recently Eick) of mysterious nature. In this paper and [BGLLS, BGLLS1, we study which of these "mysterious" examples, if any, might qualify as "standard" from the standpoint of Conjecture (8), see also SkT1, GZ]. We demystify the other examples by identifying them either as deforms of or as isomorphic to some of the "standard" examples.

1.2.1. On limited information derived from cohomology in describing deforms of Lie algebras. In \$2, we recall how deformations of Lie algebras are calculated. The trivial deformation of $\mathfrak{g}$ corresponds to the change of the basis in $\mathfrak{g}$ corresponding to a 2-coboundary, while the linear part of any global deformation is a cocycle; hence, deforms linear in the deformation parameter, also called infinitesimal deforms, correspond to cocycles representing classes of $H^{2}(\mathfrak{g} ; \mathfrak{g})$. If char $\mathbb{K}>0$, then there are "fake deformations," which means not that some of linear deforms corresponding to cocycles representing classes of $H^{2}(\mathfrak{g} ; \mathfrak{g})$ might be not extendable to a global deformation but something much worse. The textbooks and papers on Lie (super)algebra cohomology do not yet indicate the following important phenomenon:

Let each cocycle representing a class of $H^{2}(\mathfrak{g} ; \mathfrak{g}) \neq 0$ be extendable to a global deformation. This does not preclude some (or all) deforms of $\mathfrak{g}$ from being isomorphic to $\mathfrak{g}$.

Let semitrivial deformations (and their results, the deforms) be the ones whose linear parts are given by cocycles representing nontrivial cohomology classes but whose deforms are 
isomorphic to the initial Lie algebra. In addition to examples in [BLW], we show that the Jurman algebras are semitrivial deforms.

Examples of semitrivial deforms have been known to us since 1987 when we computed that ${ }^{2} \operatorname{dim} H^{2}\left(\mathfrak{o}^{\prime}(3) ; \mathfrak{o}^{\prime}(3)\right)=2$ over $\mathbb{K}$ for $p=2$ while, up to an isomorphism, there is only one simple 3-dimensional Lie algebra: $\mathfrak{o}^{\prime}(3)$. (Ten years earlier the phenomenon (9) was observed without any explanations of its origin in [DzhK.) The first explanation of the cause of the phenomenon (9) was given in [BLW]. We describe a certain type of semitrivial deformations for $p=2$ in subsec. 2.3.

1.2.2. The Vaughan-Lee algebras are not new over $\mathbb{K}$. The table on p. 948 in [Ei] shows that simple algebras of Vaughan-Lee (all new over $\mathbb{F}_{2}$ ) are only new as forms of Lie algebras known over $\mathbb{K}$ (or even over a Galois field extending $\mathbb{F}_{2}$ ).

1.2.3. The Eick algebras are new. Several simple Lie algebras were introduced in [Ei] and conjectured (e.g., because the list of "known" algebras Eick used for comparison was incomplete as compared with a wider list known to us) to be new. These algebras had to be interpreted and described in more detail than in [Ei]. With Eick's help, we recently established that all the six tentatively new algebras in [Ei] are indeed new. All the six new Eick algebras are obtained in one of the ways predicted by the conjecture [Ltow]: Eick algebras are partial Cartan prolongs (see subsec. 1.1.1), like Frank algebras for $p=3$ (cf. GL]), or deforms of something "standard."

\subsubsection{One of the Shen algebras and its generalized Cartan prolong due to Brown}

are "standard". In [Sh], Shen described several simple Lie algebras. One Shen algebra was rediscovered, together with several algebras new at that time, by Brown [Bro]. Brown's examples, described only in components in [Bro, were interpreted in [GL, BGLLS] together with a clarification of their structure and related new simple Lie superalgebras. One remarkable exceptional simple Lie algebra (we call it $\mathfrak{g s}(2)$ in honor of Shen Guangyu, who discovered it; see [Sh]) is a true analog of the Lie algebra $\mathfrak{g}(2)$ in characteristic 2. Brown rediscovered this algebra (Eick called it $\mathrm{Bro}_{2}(1,1)$ in [Ei]) and considered its Cartan prolong. We called the derived of this prolong $\mathfrak{m} \mathfrak{e}^{\prime}(5 ; \underline{N})$, and interpreted it as a version of the Melikyan algebras in characteristic 2 (see [BGLLS1]). These $\mathfrak{m e}^{\prime}(5 ; \underline{N})$, where $N_{1}=N_{2}=N_{3}=1$ are the only possible values, and its particular case $\mathfrak{g} \mathfrak{s}(2)=\mathfrak{m e}^{\prime}\left(5 ; \underline{N}_{s}\right)$ seem to be new "standard" examples. Several Shen algebras were interpreted in [BGL2] as deforms of certain "standard" algebras, and several of Shen's examples are either nonsimple or not new (see $[\mathrm{LLg}]$. Moreover, the multiplication in several Shen algebras does not satisfy the Jacobi identity, and we could not repair this.

1.2.5. Jurman and Kaplansky algebras as deforms. We started this paper intending to prove that the Jurman and Kaplansky algebras are deforms of more "conventional" simple Lie algebras (such as the two nonisomorphic versions of the Lie algebra of Hamiltonian vector fields) and their divergence-free subalgebras (see [LeP]). While this paper was being written, Grishkov published a note $3 \mathrm{GJu}$ claiming that the Jurman algebra is isomorphic to the (derived of) a Hamiltonian Lie algebra. Grishkov's paper is based on a difficult result due to Skryabin, and its claim is implicit. Consequently, we heard doubts that it is correct. It IS correct: we give an explicit isomorphism in Prop. 4.4. Amazingly, the existence of this isomorphism does not contradict the fact that the Jurman algebra is a deform corresponding to a cocycle personifying a nontrivial cohomology class of the (derived of the) Hamiltonian Lie algebra: Jurman algebras are examples of "semitrivial" deforms (see subsec. 1.2.1 and2.3.

\footnotetext{
${ }^{2}$ Hereafter, the prime' denotes the first derived, also called the commutant.

${ }^{3} \mathrm{~A}$ draft of this note was available on Grishkov's home page since 2009.
} 
In \$5, we identify type-1 Kaplansky algebras with certain known "standard" Lie algebras and prove that type-2 Kaplansky algebras are deforms of certain "standard" Lie algebras. Type-3 algebras were identified (in different terms) by Kaplansky himself as $\mathfrak{o}_{I}^{\prime}(n)$. Type-4 algebras might be "standard", see Subsection 5.2 .

1.3. Main results. The three most interesting parts of our paper:

(1) The discovery of a $\mathbb{Z} / 2$-grading quadratic in roots. Among the Lie algebras known to us, the type-2 and type-4 Kaplansky algebras are the only ones with such gradings (cf. (88)). These Kaplansky algebras are unique among the Lie algebras known to us. We present details on relations between gradings and derivations, in particular, an observation that gradings are not always defined by derivations, in Subsection [5.1.2.

(2) A method assigning a simple Lie superalgebra to every simple Lie algebra. It is illustrated with seven new series, superizations of the Kaplansky algebras in Subsection 5.3.

(3) Thanks to the insistence of a referee, we managed to give sufficient conditions for deformations, encountered so far for $p=2$, to be semitrivial (Theorem 2.3.2); but other types of semitrivial deformations are possible.

The main bulk of the paper is devoted to interpreting the simple Lie algebras discovered by Jurman and Kaplansky in terms of better known ("standard") examples of Lie algebras of Hamiltonian vector fields or their simple derived. Voluminous computations are performed using Grozman's Mathematica-based package SuperLie, cf. Lemmas 4.2.2, 6.3.1, 6.4.1, 6.4.1a,

1.4. Open problems. 1. We have described deforms of $\mathfrak{h}_{\Pi}^{\prime}(n ; \underline{N})$ for $n=2$. Investigation of the isomorphism classes of the deforms for $n>2$ and any $\underline{N}$ is a must. The classification of the deforms of the more natural nonsimple relative of the simple algebra, i.e., of $\mathfrak{h}_{\Pi}(n ; \underline{N})$, is also needed: it had led to an interpretation of previously mysterious type-2 Kaplansky algebras. The search for deforms of another relative, the Poisson Lie algebra $\mathfrak{p o}_{\Pi}(n ; \underline{N})$, is equally reasonable (answers to such problems for $p=0$ have physical interpretations), see $[\mathrm{KT}, \mathrm{KD}]$.

2. A new way to construct simple Lie algebras in the absence of classification 4 is provided in [Ei] if the structure constants belong to $\mathbb{F}_{2}$; the parametric families cannot be captured by Eick's method. Although regrettably restricted to algebras of small dimension (currently $\leq 20$ ), Eick's computer-aided approach (when its range will have been widened to dimension 250 , or at least 80) promises to give a base for a conjecture making its theoretical proof psychologically comfortable.

3. Our results show that $\mathfrak{h}_{\Pi}(2 ; \underline{N})$ and $\mathfrak{h}_{\Pi}^{\prime}(2 ; \underline{N})$ have different numbers of deforms and both types of deforms are important for classifying simple Lie algebras. The situation is similar to that in characteristic 0 , where the Lie superalgebra $\mathfrak{h}(2 n \mid m)$ has (in the only case $(2 n \mid m)=(2 \mid 2))$ more deformations than $\mathfrak{p o}(2 n \mid m)$ (see [LSh2]). A problem is to describe deformations of $\mathfrak{h}_{\Pi}(2 n ; \underline{N})$ and $\mathfrak{h}_{\Pi}^{\prime}(2 n ; \underline{N})$.

4. In [Sk], Skryabin classified nonequivalent normal shapes of the exterior 2-forms preserved by the Hamiltonian Lie algebras $\mathfrak{h}_{\Pi}(n ; \underline{N})$. It remains to classify à la Sk nonequivalent normal shapes of the tensorial 2-forms preserved by the Hamiltonian Lie algebras $\mathfrak{h}_{I}(n ; \underline{N})$ and its divergence-free subalgebras.

5. Listing all nonisomorphic deforms of $\mathfrak{g}=\mathfrak{h}_{\Pi}^{\prime}(2 ;(g, h+1))$ requires considering orbits of the $\operatorname{Aut}(\mathfrak{g})$-action on the space $H^{2}(\mathfrak{g} ; \mathfrak{g})$ following Kuznetsov and his students (see [KCh, Ch]). If the algebraic group Aut $(\mathfrak{g})$ of automorphisms of $\mathfrak{g}$ were computed, then the result in [FG] could be extended to the simple Lie algebras without a Cartan matrix. So far, this has been done only in certain particular cases (see [Pre]).

\footnotetext{
${ }^{4}$ Eick's search is random; to estimate the probability of a miss is very interesting.
} 
6. Several identification problems remain (see subsecs. 4.2.2, 5.3.3, 6.2.1, 6.5, and 6.4.1c and eq. (33) $)$.

7. A tough problem, partly solved in $\$ 2.3$, is to to give sufficient conditions for a deformation to be semitrivial.

\section{Deformations and cohomology ([BLW])}

2.1. Lie algebras. A multiparameter deformation of a Lie algebra $\mathfrak{g}$ over $\mathbb{K}$ is a Lie algebra $\mathfrak{g}_{t}$, where $t=\left(t_{1}, \ldots, t_{r}\right)$, given by a Lie algebra structure on the tensor product $\mathfrak{g} \otimes_{\mathbb{K}} \mathbb{K}[[t]]$ such that the Lie algebra $\mathfrak{g}_{0}$, i.e., the algebra obtained for $t=0$, is isomorphic to $\mathfrak{g}$. For any $x, y \in \mathfrak{g}$, the deformed bracket has the form

$$
\begin{aligned}
{[x, y]_{t_{1}, \ldots, t_{r}}=} & c^{0}(x, y)+t_{1} c_{1}^{1}(x, y)+\ldots+t_{r} c_{r}^{1}(x, y) \\
& +t_{1}^{2} c_{1,1}^{2}(x, y)+t_{1} t_{2} c_{1,2}^{2}(x, y) \ldots+t_{r}^{2} c_{r, r}^{2}(x, y)+\ldots,
\end{aligned}
$$

where $c^{0}(x, y):=[x, y]$. By linearity, it suffices to specify the deformed bracket of elements in $\mathfrak{g}$. The degree- 1 conditions say that the maps $c_{i}^{1}: \mathfrak{g} \otimes_{\mathbb{K}} \mathfrak{g} \longrightarrow \mathfrak{g}$ must be antisymmetric and must be 2-cocycles, i.e., for all $i=1, \ldots, r$ and any $x, y, z \in \mathfrak{g}$, we have

$$
\begin{aligned}
d c_{i}^{1}(x, y, z):= & c_{i}^{1}([x, y], z)+c_{i}^{1}([y, z], x)+c_{i}^{1}([z, x], y) \\
& -\left[x, c_{i}^{1}(y, z)\right]-\left[y, c_{i}^{1}(z, x)\right]-\left[z, c_{i}^{1}(x, y)\right]=0 .
\end{aligned}
$$

For brevity, we recall properties of deformations for 1-parameter deformations; the multidimensional case is considered routinely. For example, eq. (10) becomes

$$
[x, y]_{t}=c^{0}(x, y)+t c^{1}(x, y)+t^{2} c^{2}(x, y)+\ldots
$$

Two (formal) 1-parameter deforms $\mathfrak{g}_{t}$ and $\tilde{\mathfrak{g}}_{t}$ given by the collections $c=\left(c^{1}, c^{2}, \ldots\right)$ and $\tilde{c}=\left(\tilde{c}^{1}, \tilde{c}^{2}, \ldots\right)$ lead to equivalent deforms (i.e., $\mathfrak{g}_{t}$ and $\tilde{\mathfrak{g}}_{t}$ are isomorphic as Lie algebras by an isomorphism of the form $\tau(x ; t)=\sum_{i \geq 0} \tau_{i}(x) t^{i}$, where $\tau_{0}=\mathrm{id}$, for any $\left.x \in \mathfrak{g}\right)$ if and only if $c$ and $\tilde{c}$ are related as follows (for all $n>0$, where $i, j, k \geq 0$ ):

$$
\sum_{i+j=n} \tau_{i}\left(\tilde{c}^{j}(x, y)\right)=\sum_{i+j+k=n} c^{i}\left(\tau_{j}(x), \tau_{k}(y)\right) .
$$

Any change of basis of $\mathfrak{g}$ can be included in a 1 -parameter family $\tau(\cdot ; t): \mathfrak{g} \longrightarrow \mathfrak{g}$ and regarded, naturally, as a trivial deformation. We see that the trivial deformation corresponds to $d \tau_{1}$ modulo $t^{2}$; the search for the most general multiparameter deformation of a given Lie algebra therefore begins with computing the space $H^{2}(\mathfrak{g} ; \mathfrak{g})$. Its explicit basis given by 2-cocycles (representing the classes) determines infinitesimal deformations. We then try to prolong each infinitesimal deformation to higher degrees. The Jacobi identity imposes conditions on all terms in the deformed bracket, which must be satisfied in each degree. In particular, two 1-parameter degree-1 cocycles $c^{1}$ and $\tilde{c}^{1}$ are infinitesimally equivalent (i.e., $\tau=\mathrm{id}+t \tau_{1}$ modulo $t^{2}$ ) if and only if $c^{1}-\tilde{c}^{1}=d \tau_{1}$.

Let $\mathfrak{g}_{t}$ be a 1-parameter deformation of a Lie algebra $\mathfrak{g}$ given by the collection $c=\left(c^{1}, c^{2}, \ldots\right)$. The Jacobi identity yields that the coefficient of $t^{n}$ vanishes for each $n$ :

$$
\sum_{0 \leq i, j \leq n ; i+j=n}\left(c^{i}\left(c^{j}(x, y), z\right)+\operatorname{cyclic}(x, y, z)\right)=0,
$$

where $\operatorname{cyclic}(x, y, z)$ denotes the sum of all cyclic permutations of the arguments of the expression written on the left of it. We set

$$
\begin{aligned}
& {\left[\left[c^{i}, c^{j}\right]\right](x, y, z):=c^{i}\left(c^{j}(x, y), z\right)+c^{j}\left(c^{i}(x, y), z\right)+\operatorname{cyclic}(x, y, z),} \\
& c^{k} \circ c^{k}(x, y, z):=c^{k}\left(c^{k}(x, y), z\right)+\operatorname{cyclic}(x, y, z) .
\end{aligned}
$$


The brackets $\left[\left[c^{i}, c^{j}\right]\right]$ are called Nijenhuis brackets (in differential geometry) or Massey brackets (in deformation theory). The sum (13) can be expressed as a Maurer-Cartan equation:

$$
d c^{n}=\sum_{0<i<j \leq n ; i+j=n}\left[\left[c^{i}, c^{j}\right]\right]+ \begin{cases}0 & \text { if } n=2 k+1, \\ c^{k} \circ c^{k} & \text { if } n=2 k .\end{cases}
$$

To prolong an infinitesimal deformation given by a cocycle $c^{1}$, we first compute $\left[\left[c^{1}, c^{1}\right]\right]$. If $\left[\left[c^{1}, c^{1}\right]\right]=0$, then the infinitesimal deformation satisfies the Jacobi identity and is a true deformation. If $\left[\left[c^{1}, c^{1}\right]\right] \in Z^{3}(\mathfrak{g}, \mathfrak{g})$ and $\left[\left[c^{1}, c^{1}\right]\right] \notin B^{3}(\mathfrak{g}, \mathfrak{g})$, then the infinitesimal deformation is obstructed and cannot be prolonged. If $\left[\left[c^{1}, c^{1}\right]\right]=d c^{2}$ with $c^{2} \neq 0$, then $-t^{2} c^{2}$ is the seconddegree term of the deformation. To prolong the deformation to degree 3 , we compute the Massey product $\left[\left[c^{1}, c^{2}\right]\right]$. There are the three possibilities
1) $\left[\left[c^{1}, c^{2}\right]\right]=0$,
2) $\left[\left[c^{1}, c^{2}\right]\right]=d c^{3}$ for some $c^{3} \neq 0$,
3) $\left[\left[c^{1}, c^{2}\right]\right] \neq d c^{3}$ for any $c^{3}$.

If $\left[\left[c^{1}, c^{2}\right]\right]=d c^{3}$, then $-t^{3} c^{3}$ gives the third-degree prolongation of the deformation. To go up to degree 4 , we must be able to compensate $c^{2} \circ c^{2}+\left[\left[c^{1}, c^{3}\right]\right]$ by a coboundary $d c^{4}$, and so on. The main difficulty here is that the representatives of the cohomology classes and the cochains $c^{2}, c^{3}$, etc., are not uniquely 5 defined. A good choice of cochains may considerably facilitate computations. The following lemma is helpful.

2.1.1. Grozman's lemma ([BLW] $)$. For any finite-dimensional Lie algebra $\mathfrak{g}$, all cochains with coefficients in the adjoint module can be expressed as sums of tensor products of the form $a \otimes \omega$, where $a \in \mathfrak{g}$ and $\omega \in \bigwedge^{\bullet}\left(\mathfrak{g}^{*}\right)$.

Lemma. For any $c=a \otimes \omega$ with $a \in \mathfrak{g}$ and $\omega \in \bigwedge^{r}\left(\mathfrak{g}^{*}\right)$, let dc denote the coboundary of $c$ in the complex with coefficients in the adjoint module, $d \omega$ be the coboundary in the complex with coefficients in the trivial module, and da be the coboundary of a $\in \mathfrak{g}$ regarded as a 0 -cochain in the complex with coefficients in the adjoint module. Then $d c=a \otimes d \omega+d a \wedge \omega$.

2.2. Lie superalgebras. In certain problems, if $p>0$, then we must replace the (formulas of) conventional cohomology in the preceding subsection with divided power ones, see BGLL.

2.3. Semitrivial deformations. In all examples we know, the deforms of $\mathfrak{g}$ corresponding to semitrivial deformations for $p=$ char $\mathbb{K}$ are isomorphic to the initial Lie algebra via an isomorphism $\tau(\cdot, t)$ given by an expression of the form (also see [BLW], where equality $\sqrt[p]{(1+t)^{i}}=1+(\sqrt[p]{t})^{i}$ should be used $)$

$$
\tau(x ; t)=x+\sum_{i \geq 1} \tau_{i}(x)(\sqrt[p]{t})^{i} \quad \text { with the } \tau_{i} \text { satisfying conditions (12). }
$$

Therefore, the semitrivial cocycle $c$, that would have been obtained for $p=0$ as the differential of a 1-cochain $C$ such that $C(x)=\frac{\partial \tau(x, t)}{\partial t}$ for any $x \in \mathfrak{g}$, cannot be thus obtained if $p>0$, because the function $\sqrt[p]{t}$ is not differentiable for $p>0$.

For any derivation $D$ of the Lie algebra $\mathfrak{g}$, let $c_{D} \in C^{2}(\mathfrak{g} ; \mathfrak{g})$ be defined by

$$
c_{D}(x, y)=[D x, D y] \text { for any } x, y \in \mathfrak{g} .
$$

It is easy to verify that $c_{D}$ is a cocycle, i.e., $d c_{D}(x, y, z)=0$ for all $x, y, z \in \mathfrak{g}$. Moreover, if $p \neq 2$, then $c_{D}=-\frac{1}{2} d\left(D^{2}\right)$, i.e., $c_{D}$ is trivial.

\footnotetext{
${ }^{5}$ If $c^{2}$ is a solution of the equation $d c^{2}=c^{1} \circ c^{1}$, then $c^{2}+$ cocycle is also a solution. The choice of a certain $c^{2}$ affects the expression of the $c^{3}$ terms. The problem is how to find a "nice" $c^{2}$ in order to have as few $c^{3}$ terms as possible and, more importantly, vanishing Massey products in degrees $>3$. If we fail to achieve this with $c^{2}$, then we can $\operatorname{try}$ with $c^{3}$, and so on.
} 
Here is a general description of semitrivial deformations for $p=2$ in the case where an isomorphism between the algebra and its deform is a polynomial in $\sqrt{t}$. We do not know how to characterize arbitrary semitrivial deformations.

2.3.1. Lemma. Let $\mathfrak{g}$ be a Lie algebra in characteristic 2 , and let $c \in Z^{2}(\mathfrak{g} ; \mathfrak{g})$ generate a semitrivial deformation such that the isomorphism between the algebra and its deform is polynomial in $\sqrt{t}$. Then there is a derivation $D$ of $\mathfrak{g}$ such that $c$ is equivalent to $c_{D}$, i.e., $c-c_{D} \in B^{2}(\mathfrak{g} ; \mathfrak{g})$.

Proof. Let the isomorphism be $F_{t}(x)=x+\sum_{i \geq 1} f_{i}(x)(\sqrt{t})^{i}$, where only a finite number of the $f_{i} \in C^{1}(\mathfrak{g} ; \mathfrak{g})$ are nonzero. Then the deformed bracket has the form

$$
\begin{aligned}
{[x, y]_{t}=} & {[x, y]+\left(f_{1}([x, y])+\left[f_{1}(x), y\right]+\left[x, f_{1}(y)\right]\right) \sqrt{t} } \\
& +\left(\left[f_{1}(x), f_{1}(y)\right]+d f_{2}(x, y)\right) t+\text { higher order terms in } \sqrt{t}
\end{aligned}
$$

Because the coefficient of $\sqrt{t}$ is zero and the coefficient of $t$ is $c(x, y)$, we see that $f_{1}$ is a derivation, and $c$ is equivalent to $c_{f_{1}}$.

We do not know whether every cocycle of the form (17) generates a global deformation, nor do we know whether all such deformations are semitrivial or trivial. We can prove it for two classes of derivations.

2.3.2. Theorem. Let $\mathfrak{g}$ be a Lie algebra over a perfect field $\mathbb{K}$ of characteristic 2 , and let $D$ be a derivation of $\mathfrak{g}$ satisfying at least one of the following two conditions:

1. $D^{2}=\alpha D$ for some $\alpha \in \mathbb{K}$ (if $\alpha=1$, then such $D$ corresponds to a $\mathbb{Z} / 2$-grading);

2. $D$ is nilpotent.

Then the cocycle $c_{D}$ (see Lemma 2.3.1) is either trivial or semitrivial.

Proof. To prove that $c$ is either trivial or semitrivial, we show that there is a polynomial family of invertible maps $F_{t}(x)=x+\sum_{i \geq 1} f_{i}(x) t^{i}$, where only a finite number of the $f_{i} \in$ $C^{1}(\mathfrak{g} ; \mathfrak{g})$ are nonzero, such that

$$
\left[F_{t}(x), F_{t}(y)\right]=F_{t}\left([x, y]+\sum_{j \geq 1} c_{j}(x, y) t^{2 j}\right) \quad \text { for all } x, y \in \mathfrak{g}
$$

where only a finite number of the $c_{j} \in C^{2}(\mathfrak{g} ; \mathfrak{g})$ are nonzero, and $c_{1}=c_{D}$. This would mean that there exists a global deformation of $\mathfrak{g}$ given by the bracket

$$
[x, y]_{t}=[x, y]+\sum_{j \geq 1} c_{j}(x, y) t^{j}
$$

all the deformed algebras being isomorphic to $\mathfrak{g}$ with the isomorphism given by $F_{\sqrt{t}}$.

1. If $D^{2}=\alpha D$, then we set $F_{t}(x)=x+t D(x)$; this map is invertible if $t \alpha \neq 1$. We also set $c_{1}=c_{D}$ and $c_{j}=0$ for $j>1$. It is easy to see that (19) holds because $D[D x, D y]=0$.

2. Now let $D$ be nilpotent. We set

$$
f_{i}= \begin{cases}D^{i} & \text { if } i=2^{k} \text { for some } k \\ 0 & \text { otherwise }\end{cases}
$$


We note that all the $f_{i}$ are derivations. We set6

$$
c_{j}(x, y)= \begin{cases}\sum_{1 \leq s \leq 2 j-1}\left[D^{s} x, D^{2 j-s} y\right] & \text { if } j=2^{k} \text { for some } k \\ 0 & \text { otherwise }\end{cases}
$$

Because $D$ is nilpotent, the map $F_{t}$ is invertible. We must prove that the coefficients of $t^{n}$ in (19) are the same for any $n>0$, i.e., that

$$
\begin{aligned}
& {\left[f_{n}(x), y\right]+\left[x, f_{n}(y)\right]+\sum_{1 \leq i \leq n-1}\left[f_{i}(x), f_{n-i}(y)\right]} \\
& =f_{n}([x, y])+ \begin{cases}c_{m}(x, y)+\sum_{1 \leq j \leq m-1} f_{n-2 j}\left(c_{j}(x, y)\right) & \text { if } n=2 m \\
\sum_{1 \leq j \leq m} f_{n-2 j}\left(c_{j}(x, y)\right) & \text { if } n=2 m+1 .\end{cases}
\end{aligned}
$$

Because $f_{n}$ is a derivation, eq. (20) can be simplified to

$$
\sum_{1 \leq i \leq n-1}\left[f_{i}(x), f_{n-i}(y)\right]= \begin{cases}c_{m}(x, y)+\sum_{1 \leq j \leq m-1} f_{n-2 j}\left(c_{j}(x, y)\right) & \text { if } n=2 m, \\ \sum_{1 \leq j \leq m} f_{n-2 j}\left(c_{j}(x, y)\right) & \text { if } n=2 m+1 .\end{cases}
$$

If $n=1$ or if $n$ cannot be represented as a sum of at most two powers of 2 , then both sides of (21) are equal to 0 because $f_{i}$ and $c_{i}$ are only nonzero if $i$ is a power of 2 . Therefore, (21) has nonzero terms only if $n$ is the sum of at most two powers of 2 .

If $n=2^{k}+2^{l}$, where $k>l \geq 1$, then the right-hand side of (21) is

$$
\begin{aligned}
f_{2^{k}}\left(c_{2^{l-1}}(x, y)\right)+f_{2^{l}}\left(c_{2^{k-1}}(x, y)\right) & \\
= & \sum_{1 \leq s \leq 2^{l}-1}\left(\left[D^{2^{k}+s} x, D^{2^{l}-s} y\right]+\left[D^{s} x, D^{2^{k}+2^{l}-s} y\right]\right) \\
& +\sum_{1 \leq s \leq 2^{k}-1}\left(\left[D^{2^{l}+s} x, D^{2^{k}-s} y\right]+\left[D^{s} x, D^{2^{k}+2^{l}-s} y\right]\right) \\
& =\left[D^{2^{l}} x, D^{2^{k}} y\right]+\left[D^{2^{k}} x, D^{2^{l}} y\right]
\end{aligned}
$$

and the last expression is exactly the left-hand side of (21).

If $n=2^{k}+1$, where $k \geq 1$ (this case is in fact a particular subcase of the above case for $l=1$, but we consider it separately because there is no $c_{2^{l-1}}$ in this case), then the right-hand side of (21) is

$$
\begin{aligned}
f_{1}\left(c_{2^{k-1}}(x, y)\right) & =\sum_{1 \leq s \leq 2^{k}-1}\left(\left[D^{s+1} x, D^{2^{k}-s} y\right]+\left[D^{s} x, D^{2^{k}+1-s} y\right]\right) \\
& =\left[D x, D^{2^{k}} y\right]+\left[D^{2^{k}} x, D y\right]
\end{aligned}
$$

and the last expression is exactly the left-hand side of (21).

\footnotetext{
${ }^{6}$ It might be wondered, "Why does the deformed bracket satisfy the Jacobi identity? In particular, why do the $c_{j}$ satisfy the Maurer-Cartan equations?" We forget for a moment that $\mathfrak{g}$ is a Lie algebra and regard it just as an algebra. Then if (19) holds (and we show that it does), then the deformed algebra is isomorphic to $\mathfrak{g}$ with the isomorphism given by $F$. Because $\mathfrak{g}$ is a Lie algebra, the deformed algebra is also a Lie algebra, i.e., the deformed bracket satisfies Jacobi identity.
} 
If $n=2^{k}$, where $k \geq 2$, then the right-hand side of (21) is

$$
\begin{array}{r}
f_{2^{k-1}}\left(c_{2^{k-2}}(x, y)\right)+c_{2^{k-1}}(x, y) \\
=\sum_{1 \leq s \leq 2^{k-1}-1}\left(\left[D^{2^{k-1}+s} x, D^{2^{k-1}-s} y\right]+\left[D^{s} x, D^{2^{k}-s} y\right]\right)+\sum_{1 \leq s \leq 2^{k}-1}\left[D^{s} x, D^{2^{k}-s} y\right] \\
=\left[D^{2^{k-1}} x, D^{2^{k-1}} y\right],
\end{array}
$$

and the last expression is exactly the left-hand side of (21).

Finally, if $n=2$, then the left-hand side of (21) is $[D x, D y]$, same as its right side, $c_{1}(x, y)$.

\section{Modular vectorial Lie Algebras as Deforms of Each other}

Weisfeiler and Kac were the first to discover parametric families of simple finite-dimensional Lie algebras with a Cartan matrix over $\mathbb{K}$ (see [WK]). For further examples of deforms of simple Lie algebras, see [DzhK, Dzh, Sk, KD, KuJa, GL, BLW, LeP].

In this section, we extend the list of such examples and also show that several nonisomorphic Poisson Lie algebras are deforms of one Lie algebra nonsimple over $\mathbb{K}$ but simple over a ring and thus resemble forms over algebraically nonclosed fields of an algebra defined over an algebraically closed field. We regard expressions of the form $k \bmod p$, where $k \in \mathbb{Z}$, as integers from the segment $[0, p-1]$ and not as elements of $\mathbb{K}$.

3.1. Lemma. We consider a linear endomorphism $\Phi_{\alpha}$, where $\alpha \in \mathbb{K}$, of the algebra $\mathcal{O}(1 ; \underline{n})$, given by the formula

$$
\Phi_{\alpha}\left(x^{(k)}\right)=\alpha^{\left[\frac{k}{p}\right]} x^{(k)},
$$

where $\left[\frac{k}{p}\right]$ denotes the integer part of $\frac{k}{p}$ and $k<p^{n}$. If $\alpha \neq 0$, then $\Phi_{\alpha}$ is an automorphism of $\mathcal{O}(1 ; \underline{n})$.

Proof. Clearly, $\Phi_{\alpha}$ is a bijection, and we need only prove that

$$
\Phi_{\alpha}\left(x^{(k)} \cdot x^{(l)}\right)=\Phi_{\alpha}\left(x^{(k)}\right) \cdot \Phi_{\alpha}\left(x^{(l)}\right),
$$

i.e.,

$$
\alpha^{\left[\frac{k+l}{p}\right]}\left(\begin{array}{c}
k+l \\
k
\end{array}\right) x^{(k+l)}=\alpha^{\left[\frac{k}{p}\right]+\left[\frac{l}{p}\right]}\left(\begin{array}{c}
k+l \\
k
\end{array}\right) x^{(k+l)} .
$$

One can see that 7

$$
\begin{array}{ll}
{\left[\frac{k+l}{p}\right]=\left[\frac{k}{p}\right]+\left[\frac{l}{p}\right] \quad \text { if }(k \bmod p)+(l \bmod p)<p,} \\
\left(\begin{array}{c}
k+l \\
k
\end{array}\right) \equiv 0(\bmod p) \quad \text { if }(k \bmod p)+(l \bmod p) \geq p,
\end{array}
$$

and the statement in the lemma hence holds in both cases.

${ }^{7}$ The thing equal to 0 in the second line of (25) is not the same as the thing equal to $\left[\frac{k}{p}\right]+\left[\frac{l}{p}\right]$ in the first line. We also note that in the first line, the equality (involving integer parts used as power degrees) is over $\mathbb{Z}$; in the second line (involving the binomial coefficient), the equality is over $\mathbb{K}$ or modulo $p$. In both lines, the residues of $k$ and $l$ modulo $p$ should be understood as integers from the segment $[0, p-1]$; then the inequalities make sense. 
We consider the endomorphism $D_{\alpha}=\Phi_{\alpha}^{-1} \circ \partial \circ \Phi_{\alpha}$ of $\mathcal{O}(1 ; \underline{n})$ given explicitly by

$$
D_{\alpha}\left(x^{(k)}\right)= \begin{cases}\partial x^{(k)} & \text { if } p \nmid k \\ \alpha \partial x^{(k)} & \text { if } p \mid k\end{cases}
$$

We define $D_{0}$ (i.e., $D_{\alpha}$ for $\alpha=0$, when $\Phi_{0}$ is not defined) using relation (26) .

We note that if we consider the isomorphism between $\mathcal{O}(1 ; \underline{n})$ and $\mathcal{O}(2 ;(1, n-1))$ given by

$$
x^{(k)} \longleftrightarrow y_{1}^{(k \bmod p)} y_{2}^{\left(\left[\frac{k}{p}\right]\right)}
$$

then $D_{0}$ on $\mathcal{O}(1 ; \underline{n})$ corresponds to $\partial_{1}$ on $\mathcal{O}(2 ;(1, n-1))$.

Similarly, in the algebra $\mathcal{O}(d ; \underline{N})$ with indeterminates $x=\left(x_{1}, \ldots, x_{d}\right)$, we can consider the map

$$
\Phi_{\alpha}\left(x^{(r)}\right)=\alpha^{\sum_{1 \leq i \leq d}\left[\frac{r_{i}}{p}\right]} x^{(r)}
$$

which is an isomorphism for $\alpha \neq 0$. The maps (here $\partial_{i}:=\partial_{x_{i}}$ )

$$
D_{\alpha, i}=\Phi_{\alpha}^{-1} \circ \partial_{i} \circ \Phi_{\alpha} \quad \text { act as } D_{\alpha, i}\left(x^{(r)}\right)= \begin{cases}\partial_{i} x^{(r)} & \text { if } p \nmid r_{i} \\ \alpha \partial_{i} x^{(r)} & \text { if } p \mid r_{i}\end{cases}
$$

We define $D_{0, i}$ using the relations (29).

3.2. Poisson Lie algebras. We consider the Lie algebra $\mathfrak{p o}_{B}(d ; \underline{N})$, where $B=\left(B_{i j}\right)$ is an alternate (the analog of antisymmetric for $p=2$ ) nondegenerate bilinear form on a $d$ dimensional space. The space of this algebra coincides with $\mathcal{O}(d ; \underline{N})$, and the Poisson bracket is defined by eq. (6) .

We consider the deformed bracket of $\mathfrak{p o}_{B}(d ; \underline{N})$ determined by the map $\Phi_{\alpha}$ on $\mathcal{O}(d ; \underline{N})$ (we note that the deformation parameter is $\alpha-1$, not $\alpha$ ):

$$
\begin{aligned}
{[F, G]_{B, \alpha}:=\Phi_{\alpha}^{-1}\left(\left[\Phi_{\alpha}(F), \Phi_{\alpha}(G)\right]\right)=\sum_{1 \leq i, j \leq d} B_{i j} \Phi_{\alpha}^{-1}\left(\partial_{i} \Phi_{\alpha}(F) \cdot \partial_{j} \Phi_{\alpha}(G)\right) } \\
\quad=\sum_{1 \leq i, j \leq d} B_{i j} \Phi_{\alpha}^{-1}\left(\partial_{i} \Phi_{\alpha}(F)\right) \cdot \Phi_{\alpha}^{-1}\left(\partial_{j} \Phi_{\alpha}(G)\right)=\sum_{1 \leq i, j \leq d} B_{i j} D_{\alpha, i} F \cdot D_{\alpha, j} G
\end{aligned}
$$

because the map $\Phi_{\alpha}$ on $\mathcal{O}(d ; \underline{N})$ for $\alpha \neq 0$ preserves the (associative and commutative) multiplication of functions 8

We now consider the Lie algebra with the bracket (30) for any $\alpha$. Because we obtained this bracket from a trivial deformation (for $\alpha \neq 0$ ), the obtained Lie algebra is isomorphic to the initial Lie algebra $\mathfrak{p o}_{B}(d ; \underline{N})$. To what is the Lie algebra for $\alpha=0$ isomorphic?

Under the isomorphism between $\mathcal{O}(d ; \underline{N})$ and $\mathcal{O}\left(2 d ;\left(1, \ldots, 1, N_{1}-1, \ldots, N_{d}-1\right)\right)$ given by the formula

$$
x_{1}^{\left(r_{1}\right)} \ldots x_{d}^{\left(r_{d}\right)} \longleftrightarrow y_{1}^{\left(r_{1} \bmod p\right)} \ldots y_{d}^{\left(r_{d} \bmod p\right)} y_{d+1}^{\left(\left[\frac{r_{1}}{p}\right]\right)} \ldots y_{2 d}^{\left(\left[\frac{r_{d}}{p}\right]\right)}
$$

\footnotetext{
${ }^{8}$ Any automorphism of the space $\mathcal{O}(d ; \underline{N})$ produces a deformed bracket, but the second equality in (30) is due the fact that $\Phi_{\alpha}^{-1}\left(\partial_{i} \Phi_{\alpha}(F) \cdot \partial_{j} \Phi_{\alpha}(\overline{G)})=\Phi_{\alpha}^{-1}\left(\partial_{i} \Phi_{\alpha}(F)\right) \cdot \Phi_{\alpha}^{-1}\left(\partial_{j} \Phi_{\alpha}(G)\right)\right.$.
} 
the operator $D_{0, i}$ on $\mathcal{O}(d ; \underline{N})$ becomes $\partial_{i}$ on $\mathcal{O}\left(2 d ;\left(1, \ldots, 1, N_{1}-1, \ldots, N_{d}-1\right)\right)$. Hence, the Lie algebra given by commutation relation (30) with $\alpha=0$ is isomorphic to

$$
\begin{aligned}
\mathfrak{p o}_{B}(d ;(1, \ldots, 1)) \otimes \mathcal{O}\left(d ;\left(N_{1}-1, \ldots, N_{d}-1\right)\right) \\
\quad \simeq \mathfrak{p o}_{B}(d ;(1, \ldots, 1)) \otimes \mathcal{O}\left(1 ;\left(N_{1}+\cdots+N_{d}-d\right)\right) \\
\quad \simeq \mathfrak{p o}_{B}(d ;(1, \ldots, 1)) \otimes \mathcal{O}(1 ;(1))^{\otimes N_{1}+\cdots+N_{d}-d} .
\end{aligned}
$$

We see from (32) that all Poisson algebras with the same number of indeterminates, the same $\sum N_{i}$, and bilinear forms $B$ equivalent over the ground field are deforms of one Lie algebra.

Conjecturally, the statement

$$
\begin{aligned}
& \text { "any vectorial Lie algebra } X(k ; \underline{N}) \text { is a deform of the tensor product } \\
& X\left(k ; \underline{N}_{s}\right) \otimes \mathcal{O} \text {, where } \mathcal{O}:=\mathcal{O}(u ; \underline{N}) \text { with an appropriate } \underline{\tilde{N}} \text { " }
\end{aligned}
$$

holds whenever the space of the Lie algebra $X(k ; \underline{N})$ can be identified with $\mathcal{O}$, or the direct sum of several copies of $\mathcal{O}$ each endowed with its extra structure of (associative) multiplication, and the bracket can be defined using only distinguished derivatives, associative and commutative multiplication of functions, and linear operations, e.g., for $\mathfrak{v e c t}$ and $\mathfrak{k}$ (cf. the proof of Theorem 5 in [Dzh] $)$.

\section{The Jurman Algebra is A SEMitrivial DeForm}

4.1. The Jurman algebra. Jurman introduced a Lie algebra over $\mathbb{F}_{2}=\{0,1\}$ that seemed to have no analog over fields $\mathbb{K}$ of characteristic $p \neq 2$ (see [Ju]) until its interpretation in GJu. Jurman constructed this algebra by, in a sense, doubling the Zassenhaus algebra, which is the derived of the Witt algebra $\mathfrak{v e c t}(1 ; \underline{N})$. Jurman therefore called his algebra the Bi-Zassenhaus algebra denoted by $B(g, h)$. But the letter $B$ is overused, and we wish to emphasize properties of the Lie algebra $B(g, h)$ that differ from the properties in which Jurman was interested. We therefore designate this algebra $\mathfrak{j}(g, h)$ in honor of Jurman. The following description (see $[\mathrm{Ju}]$ ) allows extending the ground field and considering $\mathfrak{j}(g, h)$ over $\mathbb{K}$.

Let $g \geq 2$ and $h \geq 1$ be integers. Let $\eta=2^{g}-1$ and $\varkappa=2^{g+h} \geq 8$. Taking the elements

$$
\left\{Y_{j}(t) \mid t \in\{0,1\}, j \in\{-1,0, \ldots, \varkappa-3\}\right\}
$$

as a basis in $\mathfrak{j}(g, h)$, Jurman defined the bracket by setting

$$
\left[Y_{i}(s), Y_{j}(t)\right]=b_{s, t}^{i, j} Y_{i+j+s t(1-\eta)}(s+t),
$$

where (in the next formula, the binomial coefficients and their sum are considered modulo 2 and meaningless expressions are taken to be 0; see Example 4.1.1 for further elucidations of the meaning of the binomial coefficient for $s=t=1$ )

$$
b_{s, t}^{i, j}=\left\{\begin{array}{cl}
\left(\begin{array}{c}
i+j+s t(2-\eta) \\
i+1
\end{array}\right)+\left(\begin{array}{c}
i+j+s t(2-\eta) \\
j+1
\end{array}\right) & \text { if }-1 \leq i+j+s t(2-\eta) \leq \varkappa-3 \\
0 & \text { otherwise. }
\end{array}\right.
$$

4.1.1. Example. Let $(g, h)=(2,1)$. We have $b_{1,1}^{i,-1}=\left(\begin{array}{c}i-2 \\ i+1\end{array}\right)+\left(\begin{array}{c}i-2 \\ 0\end{array}\right)$. The first summand is meaningless for any $i$ and should be understood as a 0 . The second summand makes sense for $i \geq 2$ when it is equal to 1 .

We have $b_{1,1}^{i, 0}=\left(\begin{array}{c}i-1 \\ i+1\end{array}\right)+\left(\begin{array}{c}i-1 \\ 1\end{array}\right)$. The first summand makes no sense for any $i$, and the second summand makes no sense for $i=-1,0,1$. Each of these meaningless binomial coefficients should be understood as 0 . If $i>1$, then $\left(\begin{array}{c}i-1 \\ 1\end{array}\right) \equiv i-1(\bmod 2)$. 
We have $b_{1,1}^{i, 1}=\left(\begin{array}{c}i \\ i+1\end{array}\right)+\left(\begin{array}{c}i \\ 2\end{array}\right)$ with the first summand always meaningless (hence equal to 0 ) and the second summand equal to 0 for $i<2$.

4.2. The Jurman algebra $\mathfrak{j}(\boldsymbol{g}, \boldsymbol{h})$ as a deform of $\mathfrak{h}_{\Pi}^{\prime}(\mathbf{2} ;(\boldsymbol{g}, \boldsymbol{h}+\mathbf{1}))$. To interpret the Jurman algebra $\mathfrak{j}(g, h)$ somehow, we compare it with a known simple Lie algebra. The most plausible comparison candidates can be found in [LeP], where all possible versions of Poisson Lie algebras were described in characteristic 2, as well as Lie (sub)algebras of Hamiltonian vector fields. We realize the Poisson Lie algebra $\mathfrak{p o}_{\Pi}(2 ; \underline{N})$ by generating functions (divided powers) in the two indeterminates $p$ and $q$ with the bracket

$$
\{F, G\}=\frac{\partial F}{\partial p} \frac{\partial G}{\partial q}+\frac{\partial F}{\partial q} \frac{\partial G}{\partial p} \text { for any } F, G \in \mathcal{O}(2 ; \underline{N})
$$

where $\partial_{p}$ and $\partial_{q}$ are distinguished partial derivatives.

We consider the Lie algebra of Hamiltonian vector fields $\mathfrak{h}_{\Pi}(2 ; \underline{N})=\mathfrak{p o}_{\Pi}(2 ; \underline{N}) / \mathbb{K} \cdot 1$ and its derived $\mathfrak{h}_{\Pi}^{\prime}(2 ; \underline{N})$. We keep expressing the elements of $\mathfrak{h}_{\Pi}$ and $\mathfrak{h}_{\Pi}^{\prime}$ via generating functions having in mind, by abuse of notation, their classes modulo the center of $\mathfrak{p o}_{\Pi}$.

We recall (see [LSh1]) that the Weisfeiler filtrations were initially used to describe infinitedimensional vectorial Lie (super)algebras $\mathcal{L}$ by selecting a maximal subalgebra $\mathcal{L}_{0}$ of finite codimension. Dealing with finite-dimensional algebras, we can confine ourselves to maximal subalgebras of least or "almost least" codimension. Let $\mathcal{L}_{-1}$ be the minimal $\mathcal{L}_{0}$-invariant subspace strictly containing $\mathcal{L}_{0}$. For $i \geq 1$, we set

$$
\mathcal{L}_{-i-1}=\left[\mathcal{L}_{-1}, \mathcal{L}_{-i}\right]+\mathcal{L}_{-i} \quad \text { and } \quad \mathcal{L}_{i}=\left\{D \in \mathcal{L}_{i-1} \mid\left[D, \mathcal{L}_{-1}\right] \subset \mathcal{L}_{i-1}\right\}
$$

We thus obtain a filtration,

$$
\mathcal{L}=\mathcal{L}_{-d} \supset \mathcal{L}_{-d+1} \supset \cdots \supset \mathcal{L}_{0} \supset \mathcal{L}_{1} \supset \ldots
$$

The $d$ in (39) is called the depth of $\mathcal{L}$ and of the associated graded Lie superalgebra $\mathfrak{g}=\underset{-d \leq i}{\oplus} \mathfrak{g}_{i}$, where $\mathfrak{g}_{i}=\mathcal{L}_{i} / \mathcal{L}_{i+1}$.

We let $\mathcal{L}$ denote $\mathfrak{j}(g, h)$ when considered with a Weisfeiler filtration. Eqs. (35) and (36) imply that assuming $g, h=\infty$, we have only one maximal subalgebra of finite codimension:

$$
\mathcal{L}_{0}=\operatorname{Span}\left(Y_{i}(0), Y_{j}(1) \mid i, j \geq 0\right) .
$$

Its maximality follows from table (47). The Weisfeiler filtration corresponding to the pair $\left(\mathcal{L}, \mathcal{L}_{0}\right)$ is

$$
\mathcal{L}=\mathcal{L}_{-1} \supset \mathcal{L}_{0} \supset \mathcal{L}_{1} \supset \mathcal{L}_{2} \ldots, \quad \text { where } \mathcal{L}_{i+1}=\left\{X \in \mathcal{L}_{i} \mid[\mathcal{L}, X] \subset \mathcal{L}_{i}\right\} .
$$

Let $\operatorname{gr} \mathfrak{j}(g, h)=\oplus \mathfrak{g}_{i}$, where $\mathfrak{g}_{i}=\mathcal{L}_{i} / \mathcal{L}_{i+1}$ for $i \geq-1$.

4.2.1. Proposition. We have gr $\mathfrak{j}(g, h) \cong \mathfrak{h}_{\Pi}^{\prime}(2 ;(g, h+1))$.

Proof. For brevity, we set $\mathfrak{h}:=\mathfrak{h}_{\Pi}^{\prime}(2 ;(g, h+1))$. We first note that every element of the Cartan prolong is uniquely determined by its brackets with the elements of the $(-1)$ st component. In particular, any element $X=p^{(\beta)} q^{(\gamma)} \in \mathfrak{h}$ is uniquely determined by the conditions

$$
\begin{array}{lll}
\left(\operatorname{ad}_{p}\right)^{\gamma}\left(\operatorname{ad}_{q}\right)^{\beta-1}(X)=p, & \left(\operatorname{ad}_{p}\right)^{\gamma-1}\left(\operatorname{ad}_{q}\right)^{\beta}(X)=q & \text { for } \beta \gamma>0, \\
\left(\operatorname{ad}_{q}\right)^{\beta-1}(X)=p, & \operatorname{ad}_{p}(X)=0 & \text { for } \beta>1, \gamma=0, \\
\left(\operatorname{ad}_{p}\right)^{\gamma-1}(X)=q, & \operatorname{ad}_{q}(X)=0 & \text { for } \beta=0, \gamma>1 .
\end{array}
$$

We now pass to $\mathfrak{g}:=\operatorname{gr} \mathfrak{j}(g, h)$. Let $\bar{X}$ be the image of an arbitrary element $X \in \mathfrak{j}(p, q)$ in $\mathfrak{g}$. The definition of a filtration implies that $\operatorname{dim} \mathfrak{g}_{-1}=2$ and $\mathfrak{g}_{-1}=\operatorname{Span}\left(\overline{Y_{-1}(0)}, \overline{Y_{-1}(1)}\right)$. 
We identify

$$
\overline{Y_{-1}(0)} \longleftrightarrow q, \quad \overline{Y_{-1}(1)} \longleftrightarrow p .
$$

Let $0 \leq \alpha \leq 2^{h}-1$ and $0 \leq \beta \leq \eta=2^{g}-1$. Our next goal is to establish the correspondence

$$
\overline{Y_{i}(s)} \longleftrightarrow p^{(\beta)} q^{(2 \alpha+1-s)}, \quad \text { where } s=0,1 \text { and } i=\alpha(\eta+1)-1-s+\beta \text {. }
$$

For manual computations, it is more convenient to consider the two cases $s=0,1$ separately:

$$
\begin{array}{ll}
\overline{Y_{a}(1)} \longleftrightarrow p^{(\beta)} q^{(2 \alpha)} & \text { for } a=\alpha(\eta+1)-2+\beta, \\
\overline{Y_{b}(0)} \longleftrightarrow p^{(\beta)} q^{(2 \alpha+1)} & \text { for } b=\alpha(\eta+1)-1+\beta .
\end{array}
$$

Let $A_{\gamma, \delta}:=\left(\operatorname{ad}_{Y_{-1}(0)}\right)^{\gamma}\left(\operatorname{ad}_{Y_{-1}(1)}\right)^{\delta}$. Clearly, the image $\bar{X}$ of $X \in \mathfrak{j}(p, q)$ belongs to $\mathfrak{g}_{k}$ if and only if there exist $\gamma$ and $\delta$ such that $\gamma+\delta=k+1$ and $A_{\gamma, \delta}(X) \notin \mathcal{L}_{0}$ while for $\gamma$ and $\delta$ such that $\gamma+\delta<k+1$, we have $A_{\gamma, \delta}(X) \in \mathcal{L}_{0}$.

We now consider the brackets in the Lie algebra $\mathfrak{j}(p, q)$ :

$$
\begin{aligned}
& {\left[Y_{-1}(0), Y_{j}(s)\right]= \begin{cases}Y_{j-1}(s) & \text { if } j \geq 0, \\
0 & \text { if } j=-1,\end{cases} } \\
& {\left[Y_{-1}(1), Y_{j}(0)\right]= \begin{cases}Y_{j-1}(1) & \text { if } j \geq 0, \\
0 & \text { if } j=-1,\end{cases} } \\
& {\left[Y_{-1}(1), Y_{j}(1)\right]= \begin{cases}0 & \text { if } j<\eta-1, \\
Y_{j-\eta}(0) & \text { if } j \geq \eta-1 .\end{cases} }
\end{aligned}
$$

Equations (44) for the elements $X \in \mathfrak{j}(p, q)$ of the form $X=Y_{\beta-2}(1)$ for $2 \leq \beta \leq \eta$ imply that

$$
A_{\beta-1,0}(X)=Y_{-1}(1) \longleftrightarrow p, \quad A_{0,1}(X)=0 .
$$

Expressions (45) mean that $X \in \mathfrak{g}_{\beta-2}$, and the element $\bar{X}$ corresponds to $p^{(\beta)} \in \mathfrak{h}$. We have thus obtained the first correspondence in (43) for $\alpha=0$.

Similarly, for $X=Y_{\beta-1}(0)$, where $1 \leq \beta \leq \eta$, we have

$$
A_{\beta, 0}(X)=Y_{-1}(0) \longleftrightarrow q \text { and } A_{\beta-1,1}(X)=Y_{-1}(1) \Longrightarrow X \in \mathfrak{g}_{\beta-1} \text { and } X \longleftrightarrow p^{\beta} q \in \mathfrak{h},
$$

implying the second correspondence in eq. (43) for $\alpha=0$.

Equations (44) also imply that

$$
\left(\operatorname{ad}_{Y_{-1}(1)}\right)^{2}\left(Y_{j}(s)\right)= \begin{cases}Y_{j-\eta-1}(1) & \text { if } j \geq \eta \\ 0 & \text { if } j<\eta\end{cases}
$$

Therefore, for any $\alpha>0$ and $\beta>0$ and $X=Y_{\alpha(\eta+1)+\beta-2}(1)$, we have

$$
\begin{aligned}
& \left(\operatorname{ad}_{Y_{-1}(0)}\right)^{\beta-1}\left(\operatorname{ad}_{Y_{-1}(1)}^{2}\right)^{\alpha}(X)=A_{\beta-1,2 \alpha}(X)=Y_{-1}(1) \longleftrightarrow p, \\
& \left(\operatorname{ad}_{Y_{-1}(0)}\right)^{\beta}\left(\operatorname{ad}_{Y_{-1}(1)}^{2}\right)^{\alpha-1} \operatorname{ad}_{Y_{-1}(1)}(X)=A_{\beta, 2 \alpha-1}(X)=Y_{-1}(0) \longleftrightarrow q,
\end{aligned}
$$

implying the correspondence $\bar{X} \longleftrightarrow p^{(\beta)} q^{(2 \alpha)}$. This provides the first correspondence in eq. (43) for the case where $\alpha>0$ and $\beta>0$. We obtain the second correspondence in eq. (43) for the case where $\alpha>0$ and $\beta>0$ absolutely analogously.

It remains to consider the case where $\alpha>0$ and $\beta=0$. Let $X=Y_{\alpha(\eta+1)-1}(0)$. Then

$$
\text { Since } A_{0,2 \alpha}(X)=Y_{-1}(0) \longleftrightarrow q \text {, it follows that } \bar{X} \in \mathfrak{g}_{2 \alpha-1} \text {. }
$$

But

$$
\left[Y_{-1}(0), X\right]=Y_{\alpha(\eta+1)-2}(0)=Y_{(\alpha-1)(\eta+1)+(\eta-1)}(0) \longleftrightarrow p^{(\eta)} q^{(2 \alpha-1)} \in \mathfrak{g}_{\eta+2 \alpha-3}
$$


Because $\eta \geq 3$, it follows that $2 \alpha-1<\eta+2 \alpha-3$, and hence $\left[\overline{Y_{-1}(0)}, \bar{X}\right]=0$, i.e., the element $X$ corresponds to $q^{(2 \alpha+1)}$. This provides the second correspondence in eq. (43) for $\alpha>0$ and $\beta=0$. The first correspondence in eq. (43) for this case is obtained similarly.

We see that the maximal power of $p$ is equal to $\eta=2^{g}-1$, and hence $\underline{N}(p)=g$. Because $2 \alpha+1 \leq 2^{h+1}-1$, it follows that $\underline{N}(q)=h+1$.

Accordingly, the basis elements of the components of the first five degrees are as follows:

(47)

\begin{tabular}{|c|c|c|c|c}
\hline $\mathfrak{g}_{-1}$ & $\mathfrak{g}_{0}$ & $\mathfrak{g}_{1}$ & $\mathfrak{g}_{2}$ & $\mathfrak{g}_{3}$ \\
\hline$p \longleftrightarrow \overline{Y_{-1}(1)}$ & $p^{(2)} \longleftrightarrow \overline{Y_{0}(1)}$ & $p^{(3)} \longleftrightarrow \overline{Y_{1}(1)}$ & $p^{(4)} \longleftrightarrow \overline{Y_{2}(1)}$ & $p^{(5)} \longleftrightarrow \overline{Y_{3}(1)}$ \\
$q \longleftrightarrow \overline{Y_{-1}(0)}$ & $p q \longleftrightarrow \overline{Y_{0}(0)}$ & $p^{(2)} q \longleftrightarrow \overline{Y_{1}(0)}$ & $p^{(3)} q \longleftrightarrow \overline{Y_{2}(0)}$ & $p^{(4)} q \longleftrightarrow \overline{Y_{3}(0)}$ \\
& $q^{(2)} \longleftrightarrow \overline{Y_{\eta-1}(1)}$ & $p q^{(2)} \longleftrightarrow \overline{Y_{\eta}(1)}$ & $p^{(2)} q^{(2)} \longleftrightarrow \overline{Y_{\eta+1}(1)}$ & $p^{(3)} q^{(2)} \longleftrightarrow \overline{Y_{\eta+2}(1)}$ \\
& & $q^{(3)} \longleftrightarrow \overline{Y_{\eta}(0)}$ & $p q^{(3)} \longleftrightarrow \overline{Y_{\eta+1}(0)}$ & $p^{(2)} q^{(3)} \longleftrightarrow \overline{Y_{\eta+2}(0)}$ \\
& & & $q^{(4)} \longleftrightarrow \overline{Y_{2 \eta}(1)}$ & $p q^{(4)} \longleftrightarrow Y_{2 \eta+1}(1)$ \\
& & & & $q^{(5)} \longleftrightarrow \overline{Y_{2 \eta+1}(0)}$ \\
\hline
\end{tabular}

We let $\{\cdot, \cdot\}$ and $[\cdot, \cdot]$ denote the respective brackets in $\mathfrak{g}=\mathfrak{h}_{\Pi}^{\prime}(2 ;(g, h+1))$ and $\mathfrak{j}(g, h)$. Expressing the $\overline{Y_{i}(s)}$ in terms of monomials in $p$ and $q$, we see that for the simplest case $g=h+1$, the Jurman cocycle $c$ (which deforms $\mathfrak{h}_{\Pi}^{\prime}(2 ;(g, g))$ into the Jurman algebra) is (for any $F, G \in \mathcal{O}(2 ;(g, g))$ ), as direct calculations show,

$$
[F, G]=\{F, G\}+m_{c}(F, G), \quad \text { where } c=\sum_{m<n} p^{(\eta)} q^{(m+n-3)} \otimes d\left(q^{(m)}\right) \wedge d\left(q^{(n)}\right),
$$

and $m_{c}(F, G)$ (see eq. (55)) is the map corresponding to the cocycle $c$.

We call all other cocycles (which do not deform $\mathfrak{h}_{\Pi}^{\prime}(2 ;(g, g))$ to the Jurman algebra) nonJurman cocycles.

With respect to the pair of operators $\left(\operatorname{deg}_{p}(\cdot)-1, \operatorname{deg}_{q}(\cdot)-1\right)$ the weight of the cocycle $F \otimes d\left(G_{1}\right) \wedge \cdots \wedge d\left(G_{n}\right)$ for any $F, G_{1}, \ldots, G_{n} \in \mathfrak{h}_{\Pi}^{\prime}(2 ;(g, g))$ is equal to

$$
\left(\operatorname{deg}_{p}(F)-1-\sum\left(\operatorname{deg}_{p}\left(G_{i}\right)-1\right), \quad \operatorname{deg}_{q}(F)-1-\sum\left(\operatorname{deg}_{q}\left(G_{i}\right)-1\right)\right) .
$$

Hence, the Jurman cocycle has the weight $\left(2^{g},-2\right)$. By the symmetry $p \longleftrightarrow q$, there is another Jurman cocycle of weight $\left(-2,2^{g}\right)$ leading to an isomorphic Jurman algebra.

If $g \neq h+1$, then there is no symmetry $p \longleftrightarrow q$, but there still is another Jurman cocycle making $\mathfrak{h}_{\Pi}^{\prime}(2 ;(g, h+1))$ into $\mathfrak{j}(h+1, g-1)$. It is of the form

$$
c=\sum_{m<n} q^{(\theta)} p^{(m+n-3)} \otimes d\left(p^{(m)}\right) \wedge d\left(p^{(n)}\right), \text { where } \theta=2^{h+1}-1 .
$$

4.2.1a. Remark. In characteristic $p>2$, most of the cocycles representing classes of $H^{2}(\mathfrak{g} ; \mathfrak{g})$ are not integrable for the simple vectorial Lie algebras $\mathfrak{g}$ (see [Dzh]). If $p=2$, we do not know any simple Lie algebra $\mathfrak{g}$ with a nonintegrable cocycle representing a class of $H^{2}(\mathfrak{g} ; \mathfrak{g})$.

4.2.2. Lemma (For $(g, h)=(2,1),(2,2)$; it is a conjecture for generic values of $(g, h)$ ). Any linear combination of cocycles representing classes of $H^{2}(\mathfrak{g} ; \mathfrak{g})$ for $\mathfrak{g}=\mathfrak{h}_{\Pi}^{\prime}(2 ;(g, h+1))$ can be integrated to a global deform $\left(C f . \mathfrak{g}=\mathfrak{h}_{I}(2 ;(g, h+1))\right.$; see Lemma 6.4.1a).

For $g+h=g^{\prime}+h^{\prime}=K$, the Jurman algebras $\mathfrak{j}(g, h)$ and $\mathfrak{j}\left(g^{\prime}, h^{\prime}\right)$ regarded as $\mathbb{Z} / 2$-graded Lie algebras $\mathfrak{j}=\mathfrak{j}_{\overline{0}} \oplus \mathfrak{j}_{\overline{1}}$ with $\mathfrak{j}_{\overline{0}}$ spanned by the $Y_{i}(0)$ for all $i$ have these even parts isomorphic, and the odd parts, as modules over the even part, are also isomorphic. This is clear from eqs. (355) and (36). We note that the brackets of two odd elements given by Jurman's cocycles can be united into one bracket depending on as many parameters as there are partitions $K=g+h$ with $g \geq 2$ and $h \geq 1$. To see this, we consider the brackets of two "odd" elements and 
one "even" element and also consider the brackets of three "odd" elements; the statement is obvious in both cases. The obtained bracket depends linearly on all $K$ parameters.

4.3. Proposition. The Jurman algebra $\mathfrak{j}(g, h)$ is isomorphic to $\mathfrak{h}:=\mathfrak{h}_{\Pi}^{\prime}(2 ;(g, h+1))$. For $0 \leq k<2^{h+1}$ and $0 \leq l<2^{g-1}$, the isomorphism is given by the maps

$$
\begin{gathered}
Y_{2^{g-1} k+l-1}(0) \longleftrightarrow Y_{2^{g-1} k+l-1}=p^{\left(2^{g-1}+l\right)} q^{(k)}+(k+1) p^{(l)} q^{(k+1)}, \\
Y_{2^{g-1} k+l-2}(1) \longleftrightarrow Z_{2^{g-1} k+l-2}= \begin{cases}p^{\left(2^{g-1}+l\right)} q^{(k-1)}+(k+1) p^{(l)} q^{(k)} & \text { if } k>0, \\
p^{(l)} & \text { if } k=0 .\end{cases}
\end{gathered}
$$

Assuming that $q^{(m)}=0$ for $m<0$, we can express $Z$ in (52) uniformly:

$$
Z_{2^{g-1} k+l-2}=p^{\left(2^{g-1}+l\right)} q^{(k-1)}+(k+1) p^{(l)} q^{(k)} \quad \text { for all values } 0 \leq k<2^{h} .
$$

Proof. Taking into account that $(k+1) \bmod 2=0$ or 1 , we can unite correspondences (51) and (52) by considering the 1-parameter family of maps (we note that $\operatorname{ad}_{p^{(\mu+1)}}$ is a derivation of $\mathfrak{h}$ such that $\left.\operatorname{ad}_{p^{(\mu+1)}}^{2}=0\right)$ :

$$
\Phi_{t}: \mathfrak{h} \longrightarrow \mathfrak{h}, \quad F \mapsto F+t p^{(\mu)} \frac{\partial F}{\partial q}=F+t \operatorname{ad}_{p^{(\mu+1)}}(F), \quad \text { where } \mu=2^{g-1} .
$$

Let $m_{c}(F, G)$ be the map (54) corresponding to the cocycle $c$ (see (48)). Direct computations show that $\Phi_{\sqrt{t}}$ is an isomorphism of Lie algebras because

$$
\left\{\Phi_{t}(F), \Phi_{t}(G)\right\}=\Phi_{t}(\{F, G\})+t^{2} m_{c}(F, G) .
$$

4.3.1. Deforms of $\mathfrak{h}_{\Pi}^{\prime}(2 ;(\boldsymbol{g}, \boldsymbol{h}+\mathbf{1}))$ for the smallest values of $(\boldsymbol{g}, \boldsymbol{h})$. If $g=h+1$, it clearly suffices to consider only cocycles of nonnegative weight because of the symmetry $p \longleftrightarrow q$.

Let $(g, h)=(2,1)$. Here are cocycles representing a basis of the space $H^{2}(\mathfrak{g} ; \mathfrak{g})$. The Jurman cocycle $c:=c_{2^{g},-2}$ in (48) is $c_{4,-2}$ from our list (59) below.

4.4. Proposition. Here, $F, G \in \mathfrak{h}_{\Pi}^{\prime}(2 ;(g, h+1))$ are arbitrary, $\{\cdot, \cdot\}$ is the Poisson bracket of functions generating $\mathfrak{h}_{\Pi}(2 m ; \underline{N})$, and $\hbar \in \mathbb{K}$.

The following cocycles are semitrivial:

1. The Jurman cocycle $c_{2^{g},-2}($ see (48) ) represents the map

$$
p^{(\eta)}\left(\partial_{q} F \cdot \partial_{q}^{2} G+\partial_{q}^{2} F \cdot \partial_{q} G\right), \quad \text { where } \eta=2^{g}-1 \text {. }
$$

For $(g, h)=(2,1)$, the cocycle $c_{4,-2}$ (see (59)) represents the map

$$
m_{4,-2}(F, G)=p^{(3)}\left(\partial_{q} F \cdot \partial_{q}^{2} G+\partial_{q}^{2} F \cdot \partial_{q} G\right) .
$$

2. The cocycle $c_{0,-4}($ see (59) $)$ represents the map whose shape is independent of $(g, h)$ :

$$
m_{0,-4}(F, G)=\partial_{p} \partial_{q}^{2} F \cdot \partial_{q}^{3} G+\partial_{q}^{3} F \cdot \partial_{p} \partial_{q}^{2} G=\left\{\partial_{q}^{2} F, \partial_{q}^{2} G\right\} .
$$

3. The cocycle $c_{0,-2}$ (see (59)) is equivalent to the cochain that represents the map whose shape is independent of $(g, h)$ :

$$
m_{0,-2}(F, G)=\left\{\partial_{q} F, \partial_{q} G\right\}
$$

\section{The following cocycles are nontrivial:}

4. The cocycle $c_{2,0}($ see (59) $)$ is equivalent to the cochain representing the map

$$
m_{2,0}(F, G)=p^{(\eta)}\left(\partial_{q} F \cdot \partial_{p}^{2} G+\partial_{p}^{2} F \cdot \partial_{q} G\right), \quad \text { where } \eta=2^{g}-1
$$

which yields one of the filtered deforms (see [Dzh]). 
5. The cocycle $c_{-2,-2}$ (see (59)) is inherited from the quantization of the Poisson Lie algebra $\mathfrak{p o}_{\Pi}(2 ;(a, a))$ being the linear in the Planck constant part of the cocycle restricted to the subquotient $\mathfrak{h}_{\Pi}^{\prime}$ of $\mathfrak{p o}_{\Pi}$. The deformation turns $\mathfrak{h}_{\Pi}^{\prime}(2 ;(a, a))$ into $\mathfrak{p s l}\left(2^{a}\right)$ for any a.

The index of each cocycle is equal to its weight (further on, to save trees, we give full expression of only the cocycles with short expressions; the lexicographic order of summands adding up to the cocycle makes it possible to distinguish cocycles by looking at the pieces displayed; if these pieces are insufficient to interpret them, then see the $\mathrm{T}_{\mathrm{E} X f i l e}$ in arXiv and uncomment the hidden terms):

$$
\begin{aligned}
c_{4,-2}= & p^{(3)} \otimes d(q) \wedge d\left(q^{(2)}\right)+p^{(3)} q \otimes d(q) \wedge d\left(q^{(3)}\right)+p^{(3)} q^{(2)} \otimes d\left(q^{(2)}\right) \wedge d\left(q^{(3)}\right), \\
c_{0,-4}= & p \otimes d\left(p q^{(2)}\right) \wedge d\left(p q^{(3)}\right)+p \otimes d\left(q^{(3)}\right) \wedge d\left(p^{(2)} q^{(2)}\right)+q \otimes d\left(q^{(3)}\right) \wedge d\left(p q^{(3)}\right)+\ldots \\
c_{2,0}= & p^{(2)} \otimes d(p) \wedge d(q)+p q^{(2)} \otimes d(q) \wedge d\left(q^{(2)}\right)+p^{(3)} q \otimes d(q) \wedge d\left(p^{(2)} q\right) \\
& +p^{(3)} q^{(2)} \otimes d(p) \wedge d\left(p q^{(3)}\right)+p^{(3)} q^{(2)} \otimes d\left(q^{(2)}\right) \wedge d\left(p^{(2)} q\right)+p^{(2)} q^{(3)} \otimes d(q) \wedge d\left(p q^{(3)}\right), \\
c_{0,-2}= & p \otimes d(p) \wedge d\left(p q^{(3)}\right)+p \otimes d(p q) \wedge d\left(p q^{(2)}\right)+p \otimes d\left(q^{(2)}\right) \wedge d\left(p^{(2)} q\right) \\
& \quad+q \otimes d(q) \wedge d\left(p q^{(3)}\right)+q \otimes d(p q) \wedge d\left(q^{(3)}\right)+q \otimes d\left(q^{(2)}\right) \wedge d\left(p q^{(2)}\right)+\ldots \\
c_{-2,-2}= & p \otimes d\left(p q^{(2)}\right) \wedge d\left(p^{(3)} q\right)+q \otimes d\left(p q^{(2)}\right) \wedge d\left(p^{(2)} q^{(2)}\right) \\
& \quad+q \otimes d\left(q^{(3)}\right) \wedge d\left(p^{(3)} q\right)+p^{(2)} \otimes d\left(p^{(3)} q\right) \wedge d\left(p^{(2)} q^{(2)}\right)+p q \otimes d\left(p q^{(2)}\right) \wedge d\left(p^{(3)} q^{(2)}\right)+\ldots
\end{aligned}
$$

Proof. The fact that the maps $m_{w}$, where $w$ is a weight, do correspond to the cocycles $c_{w}$ as claimed is subject to a direct verification. The idea is as follows. We see that the image of $c_{4,-2}$ is always divisible by $p^{(3)}$ and that this image is nonzero only if both arguments are polynomials (divided powers) in $q$. Taking into account the weight of the cocycle and its (anti)symmetry, we seek $m_{c}$ in the form

$$
p^{(3)}\left(A\left(F \partial_{q}^{3} G+G \partial_{q}^{3} F\right)+B\left(\partial_{q} F \cdot \partial_{q}^{2} G+\partial_{q}^{2} F \cdot \partial_{q} G\right)\right), \quad \text { where } A, B \in \mathbb{K} .
$$

It turns out that for $A=0$ and $B=1$, we obtain the desired. For other cocycles, we seek the operators in the form $D_{1} F \cdot D_{2} G+D_{2} F \cdot D_{1} G$ or $p^{(3)}\left(D_{1} F \cdot D_{2} G+D_{2} F \cdot D_{1} G\right)$, where the $D_{i}$ are compositions of some derivations. We must check if any of these operators $m_{c}$ in fact matches $c$. It could be that to have a nice expression for $m_{c}$, we must replace the cocycle $c$ with a $\tilde{c}$ of the same cohomology class.

1. The semitriviality of the Jurman cocycle is explicitly proven for arbitrary $(g, h)$ in Proposition 4.3 .

2. We consider the maps $\Phi_{\hbar}(F)=F+\hbar D F$, where $D=\partial_{q}^{2}$. Because $D^{2}=0$, it follows that the corresponding deformed bracket produced by $c_{0,-4}$ is

$$
\{F, G\}_{\hbar}^{c_{0,-4}}:=\{F, G\}_{\sqrt{\hbar}}^{\Phi}
$$

3. In this case, although $D^{2} \neq 0$ for $D=\partial_{q}$, the derivation $D$ is still nilpotent, and Theorem 2.3.2 is hence applicable here.

4. In this case, the deformed bracket is equivalent to

$$
\{F, G\}_{\hbar}=\left(\partial_{p}+\hbar p^{(3)} \partial_{q}^{2}\right) F \cdot \partial_{q} G+\partial_{q} F \cdot\left(\partial_{p}+\hbar p^{(3)} \partial_{q}^{2}\right) G .
$$

5. Let the Poisson Lie algebra be realized by the Poisson bracket on the space of functions in $\vec{p}=\left(p_{1}, \ldots, p_{m}\right)$ and $\vec{q}=\left(q_{1}, \ldots, q_{m}\right)$. We consider the deformation (over the ground field $\mathbb{F}=\mathbb{C}$ or $\mathbb{R}$, physicists call it quantization) that turns the Poisson Lie algebra into the Lie algebra of differential operators with polynomial coefficients (see sect. 1.4.7 in [Fu]). The cocycle that determines quantization corresponds to the map

$$
\mathcal{Q}(F, G)=\sum_{1 \leq i \leq m} \frac{\partial^{2} F}{\partial p_{i}^{2}} \frac{\partial^{2} G}{\partial q_{i}^{2}}-\frac{\partial^{2} F}{\partial q_{i}^{2}} \frac{\partial^{2} G}{\partial p_{i}^{2}} \quad \text { for any } F, G \in \mathbb{F}[p, q]
$$


Here, we encounter an analog of quantization over $\mathbb{F}=\mathbb{K}$ for char $\mathbb{K}=2$. Let the coordinates of the shearing vector corresponding to $\vec{p}$ be the same as those corresponding to $\vec{q}$. Let ^ $F \mapsto \hat{F}$ be the map that to any monomial $F \in \mathcal{O}(\vec{p}, \vec{q} ;(\underline{N}, \underline{N}))$ ordered such that each $p_{i}$ is to the left of all the $q_{j}$ for all $i$ and $j$ assigns a differential operator obtained by the replacement $q_{i} \mapsto \hbar \partial_{p_{i}}$, where $\hbar \in \mathbb{K}$, for each $i$. All linear operators in the finitedimensional space $\mathcal{O}(\vec{p} ; \underline{N})$ are differential, and so the deformed Lie algebra is isomorphic to $\mathfrak{g l}(\mathcal{O}(\vec{p} ; \underline{N})) \simeq \mathfrak{g l}\left(2^{|\underline{N}|}\right)$, where $|\underline{N}|=\sum \underline{N}_{i}$. Clearly, the same cocycle induces a deformation of $\mathfrak{h}^{\prime}(2 m ; \underline{N})$ into $\mathfrak{p} \mathfrak{s l}\left(2^{|\underline{N}|}\right)$. For any $\hbar \neq 0$, the deforms are obviously isomorphic (use rescaling, i.e., divide by $\hbar$ ), and the commutator of differential operators is related to the Poisson bracket as

$$
\left.[\hat{F}, \hat{G}]=\{F, G\}_{P . b .}+O(\hbar) \quad \text { for any } F, G \in \mathcal{O}(\vec{p}, \vec{q} ; \underline{N}, \underline{N})\right) .
$$

For $m=1$, the weight of the cocycle part linear in $\hbar$ in the right-hand side of eq. (63) (up to a sign corresponding to the interchange $p \leftrightarrow q)$ is precisely $-(2,2)$.

For $\mathfrak{g}=\mathfrak{h}_{\Pi}^{\prime}(2 ;(g, h+1))$, where $(g, h)=(2,1)$, the cocycles considered in Proposition 4.4 represent a basis of $H^{2}(\mathfrak{g} ; \mathfrak{g})$. Because $\operatorname{dim} H^{2}(\mathfrak{g} ; \mathfrak{g})$ increases together with the coordinates of the shearing vector $(g, h)$, there are more deformations to be interpreted in the general case. It seems reasonable to switch attention from cocycles $c$ to maps $m_{c}$. Conjecturally, all non-Jurman cocycles correspond to the filtered deforms classified by Skryabin (see [Sk]) or to the quantization. This is so for $(g, h)=(2,1)$.

\section{What Kaplansky algebras are isomorphic to. Nonlinear superizations}

In 1981, Kaplansky described four types (in fact, five: the dimensions of the two cases of the type- 4 algebras differ significantly) of simple Lie algebras for $p=2$ (see [Kap2]). He described them only by means of the multiplication table. We interpret them in terms of familiar Lie algebras of vector fields.

Kaplansky defined the algebras in terms of J-systems resembling the notion of a root system. Over $\mathbb{F}_{2}$, a $J$-system $\Gamma$ in the space $V$ with a symmetric inner product $B$ is a set of nonzero vectors with the property that if $u, v \in \Gamma$ are distinct and satisfy $B(u, v)=1$, then $u+v \in \Gamma$. Given any $J$-system $\Gamma$, we construct a Lie algebra $\mathfrak{g}_{\Gamma}$ over $\mathbb{F}_{2}$ with basis elements $e_{u}$ for every $u \in \Gamma$, and the multiplication given by the expressions

$$
\left[e_{u}, e_{v}\right]= \begin{cases}B(u, v) e_{u+v} & \text { for } u, v \text { distinct and } u+v \in \Gamma \\ 0 & \text { for } u+v \notin \Gamma \text { or } u=v\end{cases}
$$

We note that the second half of the lower property in (64) is automatically satisfied if the form $B$ is alternating. Each Kaplansky algebra $\operatorname{Kap}_{i}(n)$, where $i=1,2,3,4$, has the form $\mathfrak{g}_{\Gamma}$ for some $\Gamma$.

Any algebra defined over $\mathbb{F}_{2}$ can obviously be defined over $\mathbb{K}$ by extension of the ground field. In what follows, speaking of Kaplansky algebras, we assume that such an extension is performed unless otherwise specified.

$\operatorname{Kap}_{1}(n)$ : For $n \geq 4$, let $\operatorname{dim} V=n$, and let $V$ carry a nondegenerate and nonalternate inner product $B$. Let $e^{1}, \ldots, e^{n}$ be an orthonormal basis of $V$. For $\Gamma$, we take all vectors in $V$ except 0 and $e=e^{1}+\cdots+e^{n}$, which can be invariantly described as the unique element satisfying $B(e, y)=B(y, y)$ for all $y$. 
Clearly, $B \sim I$. We recall that the brackets in $\mathfrak{h h}_{I}(V ; \underline{N}):=\left(V, \mathfrak{o}_{I}^{\prime}(V)\right)_{*, \underline{N}}(\mathfrak{l}$ is for "little" $)$ and $\mathfrak{h}_{I}(V ; \underline{N}):=\left(V, \mathfrak{o}_{I}(V)\right)_{*, \underline{N}}$ are the same (see $\left.[\mathrm{LeP}]\right)$ :

$$
\{F, G\}_{I}:=\sum_{1 \leq i \leq n} \partial_{z_{i}} F \cdot \partial_{z_{i}} G, \quad \text { where } F, G \in \mathbb{K}[z ; \underline{N}]
$$

only the stocks of generating functions of these Lie algebras differ. We make the assignment $e_{u} \longleftrightarrow \prod_{1 \leq i \leq n}\left(1+z_{i}\right)^{u_{i}}$. (We note that Kaplansky considered monomials in $X_{i}:=1+z_{i}$ instead of monomials in $z_{i}$.) We have $\operatorname{Kap}_{1}(n) \simeq \mathfrak{l h}_{I}^{\prime}(n)$ because $\operatorname{Kap}_{1}(n)$ does not contain $e$. In particular, we have an interpretation of $\mathrm{Kap}_{1}(4)$ sought, but not found, in [Ju, GJu]. Eick proved the isomorphism for $n=4$ (in different terms) in [Ei].

$\operatorname{Kap}_{2}(2 m)$ : Let $\operatorname{dim} V=2 m$, and let $V$ carry a nondegenerate and alternate inner product $\Pi$. We take all nonzero vectors in $V$. Kaplansky mentioned this algebra because it fits into the approach he suggested although this algebra has analogs for any characteristic $9>0$, and we could hence have ignored it; it is a filtered deform of $\mathfrak{h}_{\Pi}\left(2 m ; \underline{N}_{s}\right)$. If we had ignored it, then we would not have discovered a nonlinear $\mathbb{Z} / 2$-grading.

$\operatorname{Kap}_{3}(n)=\mathfrak{o}_{I}^{\prime}(n)$, as Kaplansky observed (in different terms). Kaplansky wrote "the gaps (in the set of values of $n=5,7$, and $\geq 9$ ) avoid duplication."

$\operatorname{Kap}_{4, a}(2 m)$, where $a=0$ or 1 , is a temporary notation, for lack of a better idea, for two similarly described and equally mysterious algebras of quite different dimensions. In their description, we need Arf invariants of quadratic forms. For a most lucid definition of an Arf invariant, see [Dye. In eq. (65), $a$ is the value of the Arf invariant (here, 0 or 1 ), and $B$ is short for "Big" and is reminiscent of the form $B$ (see eq. (66)).

Let $\operatorname{dim} V=2 m$, where $m \geq 3$, and let $Q$ be a nondegenerate quadratic form on $V$. We set

$$
\begin{array}{ll}
\operatorname{Kap}_{4, a}(2 m):=\mathfrak{g}_{\Gamma_{a}}(2 m) & \text { for } \Gamma_{a}=\{u \in V \mid Q(u)=1\}, \text { where } \operatorname{Arf}(Q)=a, \\
\operatorname{Kap}_{4, B}(2 m):=\mathfrak{g}_{\Gamma_{B}}(2 m) & \text { for } \Gamma_{B}=\{u \in V\},
\end{array}
$$

where the alternating bilinear form $B$ is given by the formula

$$
B(u, v)=Q(u+v)+Q(u)+Q(v) .
$$

We note that several quadratic forms $Q$, nonequivalent and with different values of the Arf invariant, in eq. (66) can produce the same bilinear form $B$. Observe that

$$
\operatorname{Kap}_{4, a}(2 m) \subset \operatorname{Kap}_{2}(2 m) \subset \operatorname{Kap}_{4, B}(2 m) .
$$

5.1. Proposition. 1. The Lie algebra $\operatorname{Kap}_{4, B}(2 \mathrm{~m})$ is isomorphic to the algebra whose space is $\mathcal{O}\left(2 m ; \underline{N}_{s}\right)$ with indeterminates $p_{i}$ and $q_{i}$ for $1 \leq i \leq m$ and the bracket

$$
[f, g]=\sum_{1 \leq i \leq m}\left(1+p_{i}\right)\left(1+q_{i}\right)\left(\partial_{p_{i}} f \cdot \partial_{q_{i}} g+\partial_{q_{i}} f \cdot \partial_{p_{i}} g\right) \text {. }
$$

2. The Lie algebra Kap $\operatorname{Kap}_{4, B}(2 m)$ is isomorphic to a deform of the Poisson algebra $\mathfrak{p o}_{\Pi}\left(2 m ; \underline{N}_{s}\right)$ with the deformed bracket

$$
[f, g]_{\hbar}=\sum_{1 \leq i \leq m}\left(1+\hbar p_{i} q_{i}\right)\left(\partial_{p_{i}} f \cdot \partial_{q_{i}} g+\partial_{q_{i}} f \cdot \partial_{p_{i}} g\right) \quad \text { for any } \hbar \neq 0
$$

\footnotetext{
${ }^{9}$ Kaplansky did not describe such algebras explicitly. Here is a description for any $p>0$ : Consider the polynomial algebra in $y_{i}:=\exp \left(x_{i}\right)$ and set $\partial_{x_{i}} y_{j}=\delta_{i j} y_{j}$ and $\left(y_{i}\right)^{p}=\exp \left(p x_{i}\right)=1$. In the space $\mathbb{K}[y]$, we introduce the Poisson bracket. Then $\operatorname{Kap}_{2}(2 m)$ is isomorphic to the quotient of the Poisson algebra modulo the ideal of constants.
} 
and

$$
\operatorname{Kap}_{4, B}(2 m) \simeq \operatorname{Kap}_{2}(2 m) \oplus \mathfrak{c}
$$

where the center $\mathfrak{c}$ is generated by constant functions.

Proof. 1. The isomorphism is given as follows. We choose a symplectic basis for the inner product $B$ in $V$. If $\left(u_{1}, \ldots, u_{2 m}\right)$ are coordinates of a vector $u \in V$ in this basis, then

$$
e_{u} \longleftrightarrow f_{u}=\left(1+p_{1}\right)^{u_{1}} \ldots\left(1+p_{m}\right)^{u_{m}}\left(1+q_{1}\right)^{u_{m+1}} \ldots\left(1+q_{m}\right)^{u_{2 m}}
$$

2. Clearly, (67) is a particular case of the bracket

$$
[f, g]_{\hbar}=\sum_{1 \leq i \leq m}\left(1+\hbar^{\prime} p_{i}\right)\left(1+\hbar^{\prime} q_{i}\right)\left(\partial_{p_{i}} f \cdot \partial_{q_{i}} g+\partial_{q_{i}} f \cdot \partial_{p_{i}} g\right) \quad \text { with } \hbar^{\prime}=1
$$

Here, the part linear in $\hbar^{\prime}$ describes a trivial deformation of $\mathfrak{p o}_{\Pi}\left(2 m ; \underline{N}_{s}\right)$ (as can be verified), and the quadratic part corresponds to (68) with $\hbar=\left(\hbar^{\prime}\right)^{2}$. This cocycle is nontrivial, as a computer-aided study shows.

The center is a direct summand because all weight spaces in $\operatorname{Kap}_{4, B}(2 m)$ are 1-dimensional, and the weight of the space generated by constants is 0 , but there are no two distinct weight vectors of the same weight.

\subsubsection{Kaplansky algebras $\operatorname{Kap}_{4, B}(2 m)$ and $\operatorname{Kap}_{4, a}(2 m)$ in convenient indetermi-}

nates. Examples of forms $Q_{a}$ with an Arf invariant equal to $a$ are

$$
\begin{aligned}
& Q_{0}(u)=\sum_{1 \leq i \leq m} u_{i} u_{m+i} \\
& Q_{1}(u)=u_{1}^{2}+u_{m+1}^{2}+\sum_{1 \leq i \leq m} u_{i} u_{m+i},
\end{aligned}
$$

We introduce operators $L_{i}$, where $i=1, \ldots, 2 m$ :

$$
L_{i}= \begin{cases}\left(1+p_{i}\right) \partial_{p_{i}} & \text { if } 1 \leq i \leq m \\ \left(1+q_{i-m}\right) \partial_{q_{i-m}} & \text { if } m+1 \leq i \leq 2 m\end{cases}
$$

Then $L_{i} f_{u}=u_{i} f_{u}$. We set $\Delta=\sum_{1 \leq i \leq m} L_{i} L_{i+m}$.

The subalgebras $\operatorname{Kap}_{4, a}(2 m) \subset \operatorname{Kap}_{4, B}(2 m)$ with bracket (67) are spanned by the nonzero elements $f_{u}$ such that $Q_{a}(u)=1$. From the definition (65), we derive the conditions that single out the subalgebras $\operatorname{Kap}_{4, a}(2 m)$ in $\operatorname{Kap}_{4, B}(2 m)$ :

$$
\begin{array}{ll}
f+\Delta f=0 & \text { for } \operatorname{Kap}_{4,0}(2 m), \\
f+\left(1+p_{1}\right) \partial_{p_{1}} f+\left(1+q_{1}\right) \partial_{q_{1}} f+\Delta f=0 & \text { for } \operatorname{Kap}_{4,1}(2 m) .
\end{array}
$$

The condition $\Delta f+f=0$ in (72) singles out the eigenvectors of $\Delta$ with the eigenvalue 1 . But

$$
\Delta f_{u}=\sum_{1 \leq i \leq m} u_{i} u_{i+m} f_{u}=Q_{0}(u) f_{u}
$$

and this eigenspace is therefore spanned by all $f_{u}$ such that $Q_{0}(u)=1$, which is exactly the image of $\mathrm{Kap}_{4,0}$.

The case of $\mathrm{Kap}_{4,1}$ is similar. For simplicity, we respectively replace $L_{1}^{2} f$ and $L_{m+1}^{2} f$ with $L_{1} f$ and $L_{m+1} f$. This is possible because $L_{i}^{2}=L_{i}$ and the $u_{i}$ only take values 0 and 1 . Indeed,

$$
L_{i}^{2} f_{u}=u_{i}^{2} f_{u}=u_{i} f_{u}=L_{i} f_{u}
$$


Kaplansky claimed (and we see that the claim obviously follows from (71)) that

$$
\operatorname{dim} \mathfrak{g}_{\Gamma_{a}}=2^{m-1}\left(2^{m}-(-1)^{a}\right)= \begin{cases}2^{m-1}\left(2^{m}-1\right) & \text { if } \operatorname{Arf}(Q)=0 \\ 2^{m-1}\left(2^{m}+1\right) & \text { if } \operatorname{Arf}(Q)=1\end{cases}
$$

We now study the structure of these algebras. It is more convenient to pass to the coordinates $x_{i}:=\left(1+p_{i}\right)$ and $y_{i}:=\left(1+q_{i}\right)$. The bracket (67) and operators (72) become

$$
[f, g]=\sum_{1 \leq i \leq m} x_{i} y_{i}\left(\partial_{x_{i}} f \cdot \partial_{y_{i}} g+\partial_{y_{i}} f \cdot \partial_{x_{i}} g\right)
$$

and

$$
\begin{array}{ll}
\left(1+\sum_{1 \leq i \leq m} x_{i} y_{i} \partial_{x_{i}} \partial_{y_{i}}\right) f=0 & \text { for } \operatorname{Kap}_{4,0}(2 m) \\
\left(1+x_{1} \partial_{x_{1}}+y_{1} \partial_{y_{1}}+\sum_{1 \leq i \leq m} x_{i} y_{i} \partial_{x_{i}} \partial_{y_{i}}\right) f=0 & \text { for } \operatorname{Kap}_{4,1}(2 m)
\end{array}
$$

For example,

$$
\begin{gathered}
\operatorname{Kap}_{4,0}(2)=\operatorname{Span}\left(x_{1} y_{1}\right), \operatorname{Kap}_{4,1}(2) \simeq \mathfrak{o}_{\Pi}^{\prime}(3) \simeq \mathfrak{v e c t}^{\prime}(1 ;(2)), \\
\operatorname{Kap}_{4,0}(4) \simeq \mathfrak{o}_{\Pi}^{\prime}(3) \oplus \mathfrak{o}_{\Pi}^{\prime}(3), \operatorname{Kap}_{4,1}(4) \simeq \operatorname{Kap}_{3}(5)=\mathfrak{o}_{\Pi}^{\prime}(5) .
\end{gathered}
$$

5.1.2. Gradings and derivations. The commutative subalgebra $\mathfrak{h}$ in the algebra $\mathfrak{d e r}(\mathfrak{g})$ of derivations of the type- 2 or type- 4 Kaplansky algebra $\mathfrak{g}$, i.e., the subalgebra $\mathfrak{h}$ that determines the $(\mathbb{Z} / 2)^{2 m}$-grading Kaplansky used to construct $\mathfrak{g}$, is not the maximal torus $\mathfrak{t}$ in $\mathfrak{d e r}(\mathfrak{g})$. Clearly, the type- 2 or type- 4 Kaplansky algebras are $(\mathbb{Z} / 2)^{2 m}$-graded by degrees modulo 2 with respect to each indeterminate $x_{i}$ and $y_{i}$; hence, $\mathfrak{h}=\operatorname{Span}\left(x_{i} \partial_{x_{i}}, y_{i} \partial_{y_{i}} \mid i=1, \ldots, m\right)$. On the other hand, there exists a $D \in \mathfrak{t}$ commuting with all elements of $\mathfrak{h}$ but not belonging to $\mathfrak{h}$. Equivalently, there exists a basis of $\mathfrak{g}$ simultaneously homogeneous with respect to the $(\mathbb{Z} / 2)^{2 m}$-grading Kaplansky used and with respect to an extra $\mathbb{Z} / 2$-grading given by $D$ (which is a second-order operator; see (75)), and this extra grading cannot be linearly expressed via the $(\mathbb{Z} / 2)^{2 m}$-grading. We explain why this situation is remarkable.

It might be thought that we should have taken the maximal torus from the very beginning. The catch is that in all cases we know, except these Kaplansky algebras, the extra grading operator "splits" some of the weight spaces of the previous grading. For each of these Kaplansky algebras, this is not the case: the weight spaces of the $(\mathbb{Z} / 2)^{2 m}$-grading are already 1-dimensional (except the weight-0 space if we consider the 2-closure of the algebra, but this weight-0 space does not split, anyway). Therefore, the weight spaces cannot be split further. Hence, it seems there is nowhere the extra grading can appear from, but it does appear.

We note that the derivation might be given by a differential operator of order $>1$ but the corresponding grading might still be "linear" in a sense. We consider the Witt Lie algebra $W_{n}$ over $\mathbb{K}=\mathbb{F}_{2^{n}}$, where $n>1$. For its basis, we take $\left\{e_{\alpha}\right\}_{\alpha \in \mathbb{K}}$ with the relations $\left[e_{\alpha}, e_{\beta}\right]=(\beta-\alpha) e_{\alpha+\beta}$. In fact, $W_{n}$ is $\operatorname{vect}(1 ;(n))$ over $\mathbb{K}$. On $W_{n}$, there is a natural grading: $\operatorname{deg}\left(e_{\alpha}\right)=\alpha$.

Now, consider a new grading: $\operatorname{deg}_{\text {new }}\left(e_{\alpha}\right)=\alpha^{2}$, which resembles the "nonlinear" gradings of Kaplansky algebras. Indeed, all weight spaces are 1-dimensional with respect to the old grading, and the new grading is expressed nonlinearly in terms of the old grading if $n>1$.

But if the new grading is regarded as $(\mathbb{Z} / 2)^{n}$-grading (recall that $\mathbb{K}=\mathbb{F}_{2^{n}}=(\mathbb{Z} / 2)^{n}$ as a vector space), then the new weight is obtained from the old weight by a linear transformation. The function $f: \alpha \mapsto \alpha^{2}$ is linear in the sense that $f(\alpha+\beta)=f(\alpha)+f(\beta)$, and it is nonlinear 
in the sense that it is not true that

$$
f(c \alpha) \neq c f(\alpha) \text { for any } c \in \mathbb{K} .
$$

The condition (77) holds only if $c=0$ or 1, i.e., for $n=1$.

5.1.2a. Gradings not given by derivations. Because $\operatorname{Hom}(\mathbb{Z} / q, \mathbb{Z} / p)=\{0\}$ for primes $q \neq p$, there is no derivation of the Skryabin algebra $\mathfrak{b y}$ that determines its $\mathbb{Z} / 4$-grading (here $p=3$ ), see GL.

5.1.3. The invariant symmetric bilinear forms. Kaplansky also claimed that each Kaplansky algebra of type 2, 3, or 4 has a nondegenerate invariant bilinear symmetric form (we call it $K$ here) and several other interesting properties whose verification "is quite routine." Unlike Kaplansky, we think that a lucid proof of these properties is also of interest. Here, we prove the existence of the invariant form $K$. The description of $K$ in presence of the alternate form $B$ is very simple:

$$
K\left(e_{u}, e_{v}\right)=\delta_{u, v}
$$

The form $K$ is invariant, i.e.,

$$
K\left(\left[e_{u}, e_{z}\right], e_{v}\right)=K\left(e_{u},\left[e_{z}, e_{v}\right]\right)
$$

because

$$
\begin{array}{ll}
\text { if } u+z \neq v, & \text { then } u \neq z+v, \text { and both sides vanish, and } \\
\text { if } u+z=v(\text { and } u=z+v), & \text { then the l.h.s. is } K\left(B(u, z) e_{v}, e_{v}\right)=B(u, z) \text { and } \\
& \text { the r.h.s. is } B(z, v)=B(z, u+z)=B(z, u) \\
& \text { because } B \text { is alternate and hence } B(z, z)=0 .
\end{array}
$$

We cannot guess how Kaplansky reasoned in the case of the nonalternate form $B$. In the case of the alternate form $B$, our argument relies on the invariant form on the Poisson Lie algebra induced by (the "desuperization" of) the Berezin integra 10

$$
K(f, g)=\int f g:=\text { the coefficient of the highest term of } f g
$$

if the Poisson algebra $\mathfrak{p o}_{\Pi}\left(n ; \underline{N}_{s}\right)$ is regarded as a "desuperization" of the Lie superalgebra $\mathfrak{p o}(0 \mid n)$, i.e., if the space of $\mathfrak{p o}(0 \mid n)$, the Grassmann superalgebra, is identified with the algebra of truncated polynomials in even indeterminates.

5.2. The restricted closures of Kaplansky algebras. Over $\mathbb{F}_{2}$, the 2-closures of $\mathfrak{g}=$

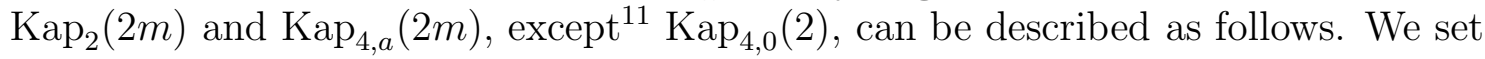

$$
[\alpha, \beta]=0, \quad\left[\alpha, e_{u}\right]=\alpha(u) e_{u} \quad \text { for any } \alpha, \beta \in V^{*}, e_{u} \in \mathfrak{g} .
$$

For a fixed $u \in V$, let $B_{u} \in V^{*}$ be the map

$$
B_{u}: v \mapsto B(u, v) \text { for any } v \in V \text {. }
$$

We can then define squaring by setting

$$
\alpha^{[2]}=\alpha, \quad e_{u}^{[2]}=B_{u} \in V^{*} .
$$

The squaring thus defined does indeed satisfy the required conditions:

$$
\left[e_{u},\left[e_{u}, e_{v}\right]\right]=\left[e_{u}, B(u, v) e_{u+v}\right]=B(u, u+v) B(u, v) e_{v}=B(u, v) e_{v}=\left[B_{u}, e_{v}\right]
$$

\footnotetext{
${ }^{10}$ See [LSh1] for a short summary of the basics of linear algebra and geometry in a super setting; for a textbook, see Lsos] or Bernstein's lectures in Del].

${ }^{11}$ This is a degenerate case: the algebra is 1-dimensional and its 2-closure is itself.
} 
and

$$
\left[\alpha,\left[\alpha, e_{u}\right]\right]=(\alpha(u))^{2} e_{u}=\alpha(u) e_{u} .
$$

Over an arbitrary field $\mathbb{K}$ of characteristic 2, the space of the 2-closure is also $\mathfrak{g} \oplus V^{*}$, but $\mathfrak{g}$ and $V^{*}$ are considered over $\mathbb{K}$, and squaring is given by the formula

$$
(a \alpha)^{[2]}=a^{2} \alpha, \quad\left(a e_{u}\right)^{[2]}=a^{2} B_{u} \in V^{*} \quad \text { for any } a \in \mathbb{K} .
$$

This description of the 2-closure shows that none of the Lie algebras $\operatorname{Kap}_{4, a}(2 m)$ for $m>2$ is isomorphic to the simple derived of the orthogonal Lie algebra of the same dimension. Indeed, the 2-closures of these algebras have different dimensions: the codimension of the simple derived of the orthogonal algebra in its 2-closure is much greater than $\operatorname{dim} V^{*}$. Because $\mathfrak{o}_{I}^{\prime}(n)$ is the algebra of zero-diagonal symmetric matrices, $\operatorname{dim} \mathfrak{o}_{I}^{\prime}(n)=\frac{1}{2} n(n-1)$.

Equation (73) implies that $\operatorname{dim} \operatorname{Kap}_{4, a}(2 m)=\operatorname{dim} \mathfrak{o}_{I}^{\prime}(n)$ if $n=2^{m}+1$ for $a=1$ or if $n=2^{m}$ for $a=0$. We therefore wonder if $\operatorname{Kap}_{4, a}(2 m)$ is a part of the $\mathfrak{o}_{I}^{\prime}(n)$ family. If $n>2$, then the 2 -closure of $\mathfrak{o}_{I}^{\prime}(n)$ is the algebra of symmetric traceless matrices, and the codimension of $\mathfrak{o}_{I}^{\prime}(n)$ in its 2 -closure is $n-1$ (the dimension of the space of diagonal matrices of trace 0 ). And from the above description, the codimension of $\mathfrak{o}_{I}^{\prime}(n)$ in its 2-closure is $\operatorname{dim} V^{*}=2 m$. Because $n-1>2 m$ (if $m>2$ ), we see that the algebras $\operatorname{Kap}_{4, a}(2 m)$ and $\mathfrak{o}_{I}^{\prime}(n)$ are nonisomorphic with the exceptions $\operatorname{Kap}_{4,0}(2) \simeq \mathfrak{o}_{I}^{\prime}(2), \operatorname{Kap}_{4,1}(2) \simeq \mathfrak{o}_{I}^{\prime}(3)$, and $\operatorname{Kap}_{4,1}(4) \simeq \mathfrak{o}_{I}^{\prime}(5)$.

5.3. General remark on superizations of Lie algebras. The basics of Lie superalgebras for $p=2$ can be found in [LeP, BGL1]. If $p=2$, there are two methods which each assigns a simple Lie superalgebra to every simple Lie algebra (see [BLLS], where it is proved that every simple Lie superalgebra is obtained by one of these two methods from a simple Lie algebra). Here, we apply one of these methods to Kaplansky algebras. Let gr be a $\mathbb{Z} / 2$ grading of $\mathfrak{g}=\mathfrak{g}_{\overline{0}} \oplus \mathfrak{g}_{\overline{1}}$ and $(\mathfrak{g}$, gr $)$ be the minimal subalgebra of $\overline{\mathfrak{g}}$ containing $\mathfrak{g}$ and all the elements $x^{[2]}$, where $x \in \mathfrak{g}_{\overline{1}}$. Clearly, there is just one way to extend the grading gr to ( $\mathfrak{g}$, gr). We define squaring by $x^{2}:=x^{[2]}$ for any $x \in \mathfrak{g}_{\overline{1}}$ and let $S(\mathfrak{g}, \mathrm{gr})$ denote the obtained Lie superalgebra. It is simple.

5.3.1. Nonlinear $\mathbb{Z} / \mathbf{2}$-gradings of Kaplansky algebras. The only known way (until this paper) to obtain a $\mathbb{Z} / 2$-grading on a Lie algebra amounts to the following. We take an arbitrary linear function of the weights, more precisely, a homomorphism from the grading group to $\mathbb{Z} / 2$. Examples of Lie superalgebras $S(\mathfrak{g}, \mathrm{gr})$ obtained from these gradings: $\mathfrak{g l}(n)$ produces $\mathfrak{g l}(k \mid n-k) ; \mathfrak{e}(6), \mathfrak{e}(7)$, and $\mathfrak{e}(8)$ produce their superizations; $\mathfrak{o}_{\Pi}(2(n+m))$ produces $\mathfrak{o}_{\Pi \Pi}(2 n \mid 2 m)$ and $\mathfrak{p e}(2 n)$ for $n=m$, whereas $\mathfrak{h}_{\Pi}(2 n ; \underline{N})$ produces $\mathfrak{h}_{\Pi}(2 k ; \underline{\tilde{N}} \mid 2 n-2 k)$ and $\mathfrak{l} \mathfrak{e}(n ; \underline{\tilde{N}})$, if the coordinates of $\underline{N}=(\underline{\tilde{N}}, 1, \ldots, 1)$ corresponding to odd indeterminates are equal to 1 (see [LeP, BGL1]).

The space $V^{*}$ (more precisely, $\mathbb{K} \otimes_{\mathbb{F}_{2}} V^{*}$, where $V^{*}$ is considered over $\mathbb{F}_{2}$ ) is a torus in the 2-closure of $\operatorname{Kap}_{2}(2 m)$ or $\operatorname{Kap}_{4, a}(2 m)$, and $u \in V$ is precisely a weight with respect to this torus. That is how we obtain what we call linear superizations of the 2-closures of $\operatorname{Kap}_{2}(2 m)$ and $\operatorname{Kap}_{4, a}(2 m)$ (see below).

The Lie algebras $\operatorname{Kap}_{2}(2 m)$ give the first (and probably unique) examples of how to introduce a $\mathbb{Z} / 2$-grading nonlinearly, and there are even two nonequivalent ways to do this.

Under any superization (linear or not), the even part of the superized Lie algebra is a Lie subalgebra of the initial Lie algebra. Hence, there is nothing extraordinary in the fact that the even part of the superized $\operatorname{Kap}_{2}(2 m) \oplus V^{*}$ is $\operatorname{Kap}_{4, a}(2 m) \oplus V^{*}$.

The whole $\operatorname{Kap}_{2}(2 m)$ cannot enter the even part of the superized Lie algebra, because the odd part would otherwise be zero. If $\mathfrak{g}=\operatorname{Kap}_{2} \oplus V^{*}$, then $V^{*}$ cannot be a part of $\mathfrak{g}_{\overline{1}}$ because $\alpha^{2}=\alpha$ for any $\alpha \in V^{*}$. Therefore, $V^{*}$ must be a part of $\mathfrak{g}_{0}$. Hence, if the whole Kap $\mathrm{K}_{2}$ goes into $\mathfrak{g}_{\overline{0}}$, there is nothing left for $\mathfrak{g}_{\overline{1}}$. 


\section{SOFIANE BOUARROUDJ ${ }^{1}$, ALEXEI LEBEDEV $^{2}$, DIMITRY LEITES ${ }^{3}$, IRINA SHCHEPOCHKINA ${ }^{4}$}

5.3.2. Linear superizations of $\operatorname{Kap}_{\mathbf{2}}(\mathbf{2 m})$ and $\operatorname{Kap}_{\mathbf{4}, \boldsymbol{a}}(\mathbf{2 m})$. Here, we say "linear" in the sense that every $e_{u}$ is homogenous and its parity is a linear function of $u \in V$ considered over $\mathbb{F}_{2}$.

We define the parity by any element $\varphi \in V^{*}$ by setting $p\left(e_{u}\right)=\varphi(u)$. Because the form $B$ is nondegenerate, there is a unique $v \in V$ such that

$$
\varphi=B_{v} \quad(\text { see (81) }) \text {, i.e., } \varphi(u)=B(v, u) \text { for all } u \in V .
$$

We let $\varphi_{v}$ denote this $\varphi$.

To show that two such superizations induced by distinct nonzero vectors $v$ and $v^{\prime}$ are isomorphic, it suffices to find a linear map $M: V \longrightarrow V$ such that

$1_{2}$. $M$ preserves $B$ for $\operatorname{Kap}_{2}(2 m)$,

14. $M$ preserves $Q$ and hence also preserves $B$ for $\operatorname{Kap}_{4, a}(2 m)$, and

2. $M v=v^{\prime}$.

The induced maps

$$
\tilde{M}: e_{u} \mapsto e_{M u}, \quad M^{*}: \varphi \mapsto \varphi \circ M^{-1} \quad \text { for any } \varphi \in V^{*}
$$

then determine an isomorphism between superizations. Indeed, for the first one,

$$
\left[\tilde{M} e_{u}, \tilde{M} e_{v}\right]=\left[e_{M u}, e_{M v}\right]=B(M u, M v) e_{M u+M v}=B(u, v) \tilde{M} e_{u+v},
$$

and if we also define $P^{\prime}\left(e_{u}\right)=B\left(v^{\prime}, u\right)$, then

$$
P^{\prime}\left(e_{M u}\right)=B\left(v^{\prime}, M u\right)=B(M v, M u)=B(v, u)=P\left(e_{u}\right) .
$$

5.3.2a. Lemma. For $K_{a p}(2 m)$, an operator $M$ with properties (85) exists for any two nonzero vectors $v$ and $v^{\prime}$ (we recall that we consider these vectors over $\mathbb{F}_{2}$ ).

Proof. If $B$ is an alternate bilinear form on a vector space $V$ of dimension $2 m$ and $B$ is nondegenerate, then there is an "alternate basis" for $B$, i.e., a basis $e^{1}, \ldots, e^{2 m}$ of $V$ such that (this is true over any field of any characteristic; see [Al])

$$
B\left(e^{i}, e^{j}\right)=\left\{\begin{aligned}
1 & \text { if } j=i+m \\
-1 & \text { if } i=j+m \\
0 & \text { in all other cases }
\end{aligned}\right.
$$

i.e., the Gram matrix of $B$ in this basis is $\left(\begin{array}{rr}0_{m} & 1_{m} \\ -1_{m} & 0_{m}\end{array}\right)$.

5.3.2b. Lemma. Let $B$ and $V$ be as in Lemma 5.3.2a and $v \in V$ be a nonzero vector. Then there is a basis $e^{1}, \ldots, e^{2 m}$ of $V$ satisfying (87) such that $e^{1}=v$.

Proof. We choose any vector $w \in V$ such that $B(v, w)=1$ and set $e^{m+1}=w$. We set

$$
V_{\perp}=\{x \in V \mid B(x, v)=B(x, w)=0\} .
$$

Then $\operatorname{dim} V_{\perp}=2 m-2$, and the restriction $B_{\perp}$ of $B$ on $V_{\perp}$ is nondegenerate. We choose $e^{2}, \ldots, e^{m}, e^{m+2}, \ldots, e^{2 m}$ as an alternate basis of $B_{\perp}$.

Now let $e^{1}, e^{2}, \ldots, e^{2 m}$ and $\tilde{e}^{1}, \tilde{e}^{2}, \ldots, \tilde{e}^{2 m}$ be two alternate bases of $V$ such that $e^{1}=v$ and $\tilde{e}^{1}=v^{\prime}$. We set $M e^{i}=\tilde{e}^{i}$. Because $B\left(M e^{i}, M e^{j}\right)=B\left(\tilde{e}^{i}, \tilde{e}^{j}\right)=B\left(e^{i}, e^{j}\right)$, it follows that $M$ preserves $B$, and $M v=v^{\prime}$.

For $\operatorname{Kap}_{4, a}(2 m)$, such an $M$ exists for two nonzero vectors $v$ and $v^{\prime}$ (considered over $\mathbb{F}_{2}$ ) if and only if $Q(v)=Q\left(v^{\prime}\right)$. Hence, there are two linear superizations for each $\operatorname{Kap}_{4, a}(2 m) \oplus V^{*}$ 
with the exception of $\operatorname{Kap}_{4, a}(2)$, where $Q(u)=1$ for any nonzero $u$, which has only one superization (it is $\left.\mathfrak{o o}_{I I}^{\prime}(1 \mid 2)\right) 12$

5.3.3. Nonlinear superizations $\operatorname{KapS}_{\mathbf{2}, \boldsymbol{a}}(\mathbf{2 m})$, and $\operatorname{KapS}_{\mathbf{4}, \boldsymbol{a}, \boldsymbol{\varepsilon}}(\mathbf{2 m})$. We note that the superization (88) is nonlinear, which means that the parity is not a linear function of $u$ because it is equal to $Q(u)+\overline{1}$. $\operatorname{set}^{13}$

$$
\begin{aligned}
\left(\operatorname{KapS}_{2, a}(2 m)\right)_{\overline{0}} & :=\operatorname{Kap}_{4, a}(2 m) \oplus V^{*} \\
\left(\operatorname{KapS}_{2, a}(2 m)\right)_{\overline{1}} & :=\operatorname{Span}\left(e_{u} \mid u \in V, u \neq 0, Q(u)=0\right)
\end{aligned}
$$

and define the bracket of even elements with any element and squaring of the odd elements by eqs. (64), (80), and

$$
\left(a e_{u}\right)^{2}:=\left(a e_{u}\right)^{[2]}=a^{2} B_{u} \in V^{*} \quad(\text { see }(\underline{83})) .
$$

Let $\operatorname{KapS}_{4, a, \varepsilon}(2 m)$ denote the nonlinear superization of $\operatorname{Kap}_{4, a}(2 m) \oplus V^{*}$ corresponding to a $v \in V$ such that $Q(v)=\varepsilon$. To describe these Lie superalgebras, we recall the definition of the parity $\varphi_{v}$ (see (84) $), \varphi_{v}(u)=B(v, u)$, and consider the following vectors $v=v_{a, \varepsilon} \in V$ assuming that the quadratic forms $Q_{a}$ are as in eq. (71):

$$
\begin{aligned}
& v_{0,0}=v_{1,1}=(1,0, \ldots, 0), \\
& v_{0,1}=(1,0, \ldots, 0,1,0, \ldots, 0) \quad(\text { the second } 1 \text { is in the }(m+1) \text { th position }), \\
& v_{1,0}=(0,1,0, \ldots, 0) \text { for } m>1 \quad \text { (if } m=1, \text { then } Q_{1}(v)=1 \text { for any nonzero } \\
& v \in V, \text { and } v_{0,1} \text { hence cannot be chosen). }
\end{aligned}
$$

We set

$$
\begin{aligned}
& \operatorname{KapS}_{4, a, \varepsilon}(2 m)_{\overline{0}}:=\operatorname{Span}\left(e_{u} \mid u \neq 0, Q_{a}(u)=1, B\left(v_{a, \varepsilon}, u\right)=0\right) \oplus V^{*}, \\
& \operatorname{KapS}_{4, a, \varepsilon}(2 m)_{\overline{1}}:=\operatorname{Span}\left(e_{u} \mid u \neq 0, Q_{a}(u)=1, B\left(v_{a, \varepsilon}, u\right)=1\right) .
\end{aligned}
$$

(Here, as usual, $B(u, v)=Q(u+v)-Q(u)-Q(v)$, and in this case $Q=Q_{a}$.)

\subsection{3a. There are no nonlinear superizations of $\operatorname{Kap}_{4, a}(2 m)$ induced by nonlinear}

superizations of $\mathbf{K a p}_{\mathbf{2}}(\mathbf{2 m})$. In $\operatorname{KapS}_{2, a}(2 m)$ corresponding to a form $Q$, we take the part corresponding to $\operatorname{Kap}_{4, a}(2 m)$ with another form $Q^{\prime}$. This is a Lie subsuperalgebra. Can we do this? We can, but this superization fortunately (the classification would otherwise certainly be a nightmare) coincides with a linear one. This subsuperalgebra is singled out by the condition $Q^{\prime}(u)=1$ while its even part is singled out by this condition together with the extra condition $Q(u)=1$, which can be replaced with $Q(u)+Q^{\prime}(u)=0$; because both $Q$ and $Q^{\prime}$ should yield the same bilinear form $B$, the quadratic form $Q+Q^{\prime}$ degenerates into a linear function. Therefore, this superization is equivalent to a linear one.

Therefore, up to an isomorphism, there is one linear superization of $\operatorname{Kap}_{2}(2 m)$, this superization 14 is here denoted by $\operatorname{KapLS}_{2}(2 m)$. The three Lie superalgebras $\operatorname{KapLS}_{2}(2 m)$ and $\operatorname{KapS}_{2, a}(2 m)$ for $a=0,1$ are nonisomorphic.

\footnotetext{
${ }^{12}$ In fact, the argument with the map (86) does not prove that the two superizations of $\operatorname{Kap}_{4, a}(2 m)$ are nonisomorphic but only that there is no isomorphism of the form (86) between them. Conjecturally, they are nonisomorphic.

${ }^{13}$ We are not sure which notation to use here. The $Q_{a}$ are just examples of quadratic forms with the Arf invariant $a$, while the $Q$ in (88) can be any quadratic form with the Arf invariant $a$.

${ }^{14}$ It would be interesting to find out if $\operatorname{KapS}_{2}(2 m)$ is a deform of a superization of $\mathfrak{h}_{\Pi}$. This is clearly not so for $\mathfrak{h}_{\Pi}(2 k \mid 2 m-2 k)$ because their dimensions differ (we recall that $\operatorname{KapS}_{2}(2 m)$ contains $V^{*}$ ). But it might be a deform of a larger algebra. Conjecturally, it is not.
} 


\section{D'INACHEVÉ}

6.1. Generalizations of the Jurman construction. We consider $\mathfrak{a}(2 ;(g, h))$, the Lie algebra whose space is $\mathcal{O}(2 ;(g+h, 1))$, and the bracket of any $F, G \in \mathcal{O}(2 ;(g+h, 1))$ is given by the formula (we write $x$ and $y$ to avoid confusion with $p$ and $q$ in the preceding sections)

$$
\begin{aligned}
{[F, G] } & =\partial_{x} F \cdot\left(\partial_{y}+y \partial_{x}^{2^{g}}\right) G+\left(\partial_{y}+y \partial_{x}^{2^{g}}\right) F \cdot \partial_{x} G \\
& =[F, G]_{P . b .}+y\left(\partial_{x} F \cdot \partial_{x}^{2^{g}} G+\partial_{x}^{2^{g}} F \cdot \partial_{x} G\right) .
\end{aligned}
$$

Both $\partial_{x}$ and $\partial_{y}+y \partial_{x}^{2^{g}}$ are derivations of $\mathcal{O}(2 ;(g+h, 1))$ and they mutually commute and therefore the Jacobi identity holds. (We note that the fact that the conventional Poisson bracket satisfies the Jacobi identity is a corollary of the similar properties of $\partial_{x}$ and $\partial_{y}$.) The first derived $\mathfrak{a}^{\prime}(2 ;(g, h))$ of $\mathfrak{a}(2 ;(g, h))$ is spanned by all monomials except the highest-degree element $x^{\left(2^{g+h}-1\right)} y$.

6.1.1. Lemma. We have $\mathfrak{a}^{\prime}(2 ;(g, h)) / \mathfrak{c} \simeq \mathfrak{j}(g, h)$ with an isomorphism realized by the expressions

$$
Y_{i}(0)=x^{(i+1)} y, \quad Y_{i}(1)=x^{(i+2)} .
$$

Proof. We directly verify the commutation relations. We first note that the brackets of $Y_{i}(0)$ with anything do not contain additional terms because these terms contain not $\partial_{y}$ but multiplication by $y$, and $Y_{i}(0)$ already contains $y$ while $y \cdot y=0$. We also note that $\left[Y_{i}(1), Y_{j}(1)\right]_{P . b .}=0$. Taking this into account, we see that

$$
\begin{aligned}
{\left[Y_{i}(0), Y_{j}(0)\right] } & =x^{(i)} y \cdot x^{(j+1)}+x^{(i+1)} \cdot x^{(j)} y \\
& =\left(\left(\begin{array}{c}
i+j+1 \\
j+1
\end{array}\right)+\left(\begin{array}{c}
i+j+1 \\
i+1
\end{array}\right)\right) x^{(i+j+1)} y \\
& =\left(\left(\begin{array}{c}
i+j+1 \\
i+1
\end{array}\right)+\left(\begin{array}{c}
i+j+1 \\
j+1
\end{array}\right)\right) Y_{i+j}(0), \\
{\left[Y_{i}(0), Y_{j}(1)\right] } & =x^{(i+1)} \cdot x p^{(j+1)}=\left(\begin{array}{c}
i+j+2 \\
i+1
\end{array}\right) x^{(i+j+2)} \\
& =\left(\left(\begin{array}{c}
i+j+1 \\
i+1
\end{array}\right)+\left(\begin{array}{c}
i+j+1 \\
j+1
\end{array}\right)\right) Y_{i+j}(1) .
\end{aligned}
$$

The statement of eq. (92) is clear; that of eq. (93) holds because if $i+j+1 \geq 0$, then

$$
\left(\begin{array}{c}
i+j+1 \\
i+1
\end{array}\right)+\left(\begin{array}{c}
i+j+1 \\
j+1
\end{array}\right)=\left(\begin{array}{c}
i+j+1 \\
i+1
\end{array}\right)+\left(\begin{array}{c}
i+j+1 \\
i
\end{array}\right)=\left(\begin{array}{c}
i+j+2 \\
i+1
\end{array}\right)
$$

while if $i+j+1<0$, then $i=j=-1$, and $\left(\begin{array}{c}i+j+2 \\ i+1\end{array}\right) x^{(i+j+2)}=1$, i.e., is a constant, which generates the center $\mathfrak{c}$. Therefore, it is equal to 0 in the quotient $\mathfrak{a}^{\prime}(2 ;(g, h)) / \mathfrak{c}$. Hence, in this case, we also have $\left(\left(\begin{array}{c}i+j+1 \\ i+1\end{array}\right)+\left(\begin{array}{c}i+j+1 \\ j+1\end{array}\right)\right) Y_{i+j}(1)=0$.

We now have

$$
\begin{aligned}
{\left[Y_{i}(1), Y_{j}(1)\right] } & =y\left(x^{(i+1)} \cdot x^{(j+1-\eta)}+x^{(i+1-\eta)} \cdot x^{(j+1)}\right) \\
& =\left(\left(\begin{array}{c}
i+j+2-\eta \\
i+1
\end{array}\right)+\left(\begin{array}{c}
i+j+2-\eta \\
j+1
\end{array}\right)\right) x^{i+j+2-\eta} y \\
& =\left(\left(\begin{array}{c}
i+j+2-\eta \\
i+1
\end{array}\right)+\left(\begin{array}{c}
i+j+2-\eta \\
j+1
\end{array}\right)\right) Y_{i+j+1-\eta}(0) .
\end{aligned}
$$

We hence see that the commutation relations are the same as in $\mathfrak{j}(g, h)$ in all cases. 
6.2. Comparison with known Lie algebras. The direct analog of bracket (91) exists in any characteristic $p$ and has the form

$$
\begin{aligned}
{[F, G] } & =\partial_{x} F \cdot\left(\partial_{y}+y^{p-1} \partial_{x}^{p^{g}}\right) G+\left(\partial_{y}+y^{p-1} \partial_{x}^{p^{g}}\right) F \cdot \partial_{x} G \\
& =[F, G]_{P . b .}+y^{p-1}\left(\partial_{x} F \cdot \partial_{x}^{p^{g}} G+\partial_{x}^{p^{g}} F \cdot \partial_{x} G\right) .
\end{aligned}
$$

For $p>3$, all finite-dimensional simple Lie algebras are classified, and this bracket is therefore the bracket of a known Lie algebra.

6.2.1. Question. To which of the filtered deforms of Lie algebras of Hamiltonian vector fields (see [LeP]) is the Lie algebra with the bracket (95) isomorphic?

6.3. On further generalizations. We can replace $\partial_{y}+y \partial_{x}^{2^{g}}$ with $\partial_{y}+R(y) \partial_{x}^{2^{g}}$, where $R$ is any polynomial of a divided degree $\leq \underline{N}(y)$. Conjecturally, the only $R$ of interest is the monomial of highest possible degree; the other shapes of $R$ can be reduced to this or a constant. But it seems that for any $\underline{N}(y)>1$, the result is $\mathfrak{j}(g+N-1, h)$ : the cocycles that make Jurman algebras from $\mathfrak{h}_{\Pi}^{\prime}(2 ;(2,2))$ and $\mathfrak{h}_{\Pi}^{\prime}(2 ;(3,2))$ change the bracket in precisely this way.

We can consider any number $k$ of pairs of indeterminates with the bracket

$$
[F, G]=\sum_{1 \leq i \leq k} \partial_{x_{i}} F \cdot\left(\partial_{y_{i}}+y_{i} \partial_{x_{i}}^{2^{g_{i}}}\right) G+\left(\partial_{y_{i}}+y_{i} \partial_{x_{i}}^{2^{g_{i}}}\right) F \cdot \partial_{x_{i}} G .
$$

We note that the $g_{i}$ can differ for different $i$.

6.3.1. Lemma. The Lie algebra $\mathfrak{a}_{\Pi}^{\prime}\left(2 k ;\left(g_{1}, h_{1}\right), \ldots,\left(g_{k}, h_{k}\right)\right)$ has no center and no homogenous ideals for $k=2$ and $\left(g_{1}, h_{1}\right)=\left(g_{2}, h_{2}\right)=(2,1)$. (Conjecturally, it is simple.)

6.4. $\mathfrak{a}_{\boldsymbol{I}}(\mathbf{2} ;(\boldsymbol{g}, \boldsymbol{h}))$. The Lie algebra $\mathfrak{a}_{I}(2 ;(g, h))$ based on $\mathfrak{h}_{I}(2 ;(g+h, 1))$ can also be generalized in the above way by beginning with the bracket

$$
[F, G]=\partial_{x} F \cdot \partial_{x} G+\left(\partial_{y}+y \partial_{x}^{2^{g}}\right) F \cdot\left(\partial_{y}+y \partial_{x}^{2^{g}}\right) G
$$

and generalizing further as indicated above.

6.4.1. Lemma. The Lie algebra $\mathfrak{a}_{I}\left(2 k ;\left(g_{1}, h_{1}\right), \ldots,\left(g_{k}, h_{k}\right)\right)$ has no center and no homogenous ideals for $k=2$ and $\left(g_{1}, h_{1}\right)=\left(g_{2}, h_{2}\right)=(2,1)$. (Conjecturally, it is simple.)

(98)

$$
\begin{aligned}
& c_{-4}^{1}=p \otimes\left(d(p q) \wedge d\left(p^{(2)} q^{(3)}\right)+d\left(p q^{(2)}\right) \wedge d\left(p^{(2)} q^{(2)}\right)+d\left(p q^{(3)}\right) \wedge d\left(p^{(2)} q\right)\right)+\ldots, \\
& c_{-4}^{2}=p \otimes d\left(p^{(2)} q\right) \wedge d\left(p^{(3)} q\right)+q \otimes d\left(p^{(3)}\right) \wedge d\left(p^{(3)} q\right)+q \otimes d\left(p^{(2)} q\right) \wedge d\left(p^{(2)} q^{(2)}\right)+\ldots, \\
& c_{-4}^{3}=p \otimes d(p q) \wedge d\left(p^{(2)} q^{(3)}\right)+p \otimes d\left(p q^{(2)}\right) \wedge d\left(p^{(2)} q^{(2)}\right)+p \otimes d\left(p q^{(3)}\right) \wedge d\left(p^{(2)} q\right)+\ldots, \\
& c_{-2}^{1}=p \otimes d\left(p^{(2)}\right) \wedge d\left(p^{(3)}\right)+q \otimes d\left(p^{(2)}\right) \wedge d\left(p^{(2)} q\right)+q^{(2)} \otimes d\left(p^{(2)}\right) \wedge d\left(p^{(2)} q^{(2)}\right)+\ldots, \\
& c_{-2}^{2}=p \otimes d\left(q^{(2)}\right) \wedge d\left(p q^{(2)}\right)+q \otimes d\left(q^{(2)}\right) \wedge d\left(q^{(3)}\right)+p^{(2)} \otimes d\left(q^{(2)}\right) \wedge d\left(p^{(2)} q^{(2)}\right)+\ldots, \\
& c_{-2}^{3}=p \otimes d\left(p^{(2)}\right) \wedge d\left(p^{(3)}\right)+q \otimes d(p) \wedge d\left(p^{(3)} q\right)+q \otimes d\left(p^{(2)}\right) \wedge d\left(p^{(2)} q\right)+\ldots, \\
& c_{-2}^{4}=p \otimes d\left(p^{(2)}\right) \wedge d\left(p q^{(2)}\right)+p \otimes d\left(p^{(3)}\right) \wedge d\left(q^{(2)}\right)+q \otimes d\left(p^{(2)}\right) \wedge d\left(q^{(3)}\right)+\ldots, \\
& \left.c_{0}=p \otimes d(q) \wedge d(p q)+p^{(2)} \otimes d(q) \wedge d\left(p^{(2)} q\right)\right)+p^{(3)} \otimes d(q) \wedge d\left(p^{(3)} q\right)+\ldots \\
& \left.c_{2}^{1}=q^{(3)} \otimes d(q) \wedge d\left(p^{(2)}\right)\right)+p q^{(3)} \otimes d(q) \wedge d\left(p^{(3)}\right)+p q^{(3)} \otimes d\left(p^{(2)}\right) \wedge d(p q)+\ldots, \\
& c_{2}^{2}=p^{(3)} \otimes d(p) \wedge d\left(q^{(2)}\right)+p^{(3)} q \otimes d(p) \wedge d\left(q^{(3)}\right)+p^{(3)} q \otimes d\left(q^{(2)}\right) \wedge d(p q)+\ldots, \\
& c_{2}^{3}=q^{(3)} \otimes d(q) \wedge d\left(q^{(2)}\right)+p q^{(3)} \otimes d(q) \wedge d\left(p q^{(2)}\right)+p q^{(3)} \otimes d\left(q^{(2)}\right) \wedge d(p q)+\ldots, \\
& c_{2}^{4}=p^{(3)} \otimes d(p) \wedge d\left(p^{(2)}\right)+p^{(3)} q \otimes d(p) \wedge d\left(p^{(2)} q\right)+p^{(3)} q \otimes d\left(p^{(2)}\right) \wedge d(p q)+\ldots, \\
& c_{6}=p^{(3)} q^{(3)} \otimes d(p) \wedge d(q) .
\end{aligned}
$$


6.4.1a. Lemma. For $\mathfrak{g}:=\mathfrak{h}_{I}(2 ;(2,2))$, each cocycle (98) representing the weight elements of $H^{2}(\mathfrak{g} ; \mathfrak{g})$ is integrable, and all except $c_{-2}^{3}$ (see eq. (99)) are linearly integrable.

Proof. Computer-aided. The nonlinear deform (cf. [BLW]) is

$$
[\cdot, \cdot]_{\hbar}=[,]+c_{-2}^{3} \hbar+A \hbar^{2}+B \hbar^{3}
$$

where

$$
\begin{aligned}
A= & p q^{(3)} \otimes d\left(p^{(2)} q^{(2)}\right) \wedge d\left(p^{(3)} q^{(3)}\right)+p q^{(3)} \otimes d\left(p^{(2)} q^{(3)}\right) \wedge d\left(p^{(3)} q^{(2)}\right) \\
& +p^{(2)} q^{(2)} \otimes d\left(p^{(3)} q\right) \wedge d\left(p^{(3)} q^{(3)}\right)+q^{(3)} \otimes d\left(p q^{(2)}\right) \wedge d\left(p^{(3)} q^{(3)}\right) \\
& +q^{(3)} \otimes d\left(p q^{(3)}\right) \wedge d\left(p^{(3)} q^{(2)}\right)+p q^{(2)} \otimes d\left(p^{(2)} q\right) \wedge d\left(p^{(3)} q^{(3)}\right) \\
& +p^{(2)} q \otimes d\left(p^{(3)} q\right) \wedge d\left(p^{(3)} q^{(2)}\right)+q^{(2)} \otimes d(p q) \wedge d\left(p^{(3)} q^{(3)}\right) \\
& +q \otimes d\left(p^{(3)}\right) \wedge d\left(p^{(3)} q\right)+q \otimes d(p q) \wedge d\left(p^{(3)} q^{(2)}\right), \\
B= & q^{(3)} \otimes d\left(p^{(3)} q^{(2)}\right) \wedge d\left(p^{(3)} q^{(3)}\right) .
\end{aligned}
$$

6.4.1b. Claim: The Lie algebra $\mathfrak{a}_{I}(2 ;(g, h))$ is a deform of $\mathfrak{h}_{I}(2 ;(\boldsymbol{g}+\boldsymbol{h}, \mathbf{1}))$. To prove this for the smallest values of $(g, h)$, we list all infinitesimal deforms of $\mathfrak{h}_{I}(2 ;(2,2))$. For the cochain $F \otimes\left(d G_{1} \wedge \cdots \wedge d G_{n}\right)$, where $F, G_{1}, \ldots, G_{n} \in \mathfrak{h}_{I}(2 ;(g+h, 1))$, its weight is equal to

$$
\left(\left(\operatorname{deg}_{p}(F)-\sum_{1 \leq i \leq n} \operatorname{deg}_{p}\left(G_{i}\right)\right) \bmod 2, \quad\left(\operatorname{deg}_{q}(F)-\sum_{1 \leq i \leq n} \operatorname{deg}_{q}\left(G_{i}\right)\right) \bmod 2\right)
$$

We note that this grading is induced by elements of a maximal torus, more specifically, by $p^{(2)}$ and $q^{(2)}$. This grading is therefore modulo 2 and is not a $\mathbb{Z}$-grading. This algebra also has the outer grading $\operatorname{deg}_{\text {out }}$ given by

$$
\operatorname{deg}(p)=\operatorname{deg}(q)=1, \quad \operatorname{deg}_{\text {out }}(F)=\operatorname{deg}(F)-2, \quad \operatorname{deg}_{\text {out }}(d F)=2-\operatorname{deg}(f) .
$$

The cocycles (102) are all of weight $\{0,0\}$. They are indexed in accordance with deg $_{\text {out }}$.

6.4.1c. Question. How to interpret the non-Jurman cocycles à la Proposition 4.4 for the other values of $(g, h)$ ? For example, for $(g, h)=(3,1)$ and $(2,2)$, i.e., for the deformations of $\mathfrak{h}_{\Pi}^{\prime}(2 ;(3,2)) \simeq \mathfrak{h}_{\Pi}^{\prime}(2 ;(2,3))$, the Jurman cocycle deforming $\mathfrak{h}_{\Pi}^{\prime}(2 ;(3,2))$ into $\mathfrak{j}(3,1)$ is $c_{-2,8}$, 
and the Jurman cocycle deforming $\mathfrak{h}_{\Pi}^{\prime}(2 ;(2,3))$ into $\mathfrak{j}(2,2)$ is $c_{4,-2}$, see (102).

$(102)$

$$
\begin{aligned}
& c_{0,-8}=p \otimes d\left(p q^{(4)}\right) \wedge d\left(p q^{(5)}\right)+p \otimes d\left(q^{(5)}\right) \wedge d\left(p^{(2)} q^{(4)}\right)+q \otimes d\left(p q^{(4)}\right) \wedge d\left(q^{(6)}\right)+\ldots, \\
& c_{1,-7}=p \otimes d\left(q^{(4)}\right) \wedge d\left(p q^{(4)}\right)+q \otimes d\left(q^{(4)}\right) \wedge d\left(q^{(5)}\right)+p^{(2)} \otimes d\left(q^{(4)}\right) \wedge d\left(p^{(2)} q^{(4)}\right)+\ldots, \\
& c_{4,-4}=p^{(3)} \otimes d(q) \wedge d\left(q^{(4)}\right)+p^{(3)} q \otimes d(q) \wedge d\left(q^{(5)}\right)+p^{(3)} q \otimes d\left(q^{(2)}\right) \wedge d\left(q^{(4)}\right)+\ldots \\
& c_{4,-2}=p^{(3)} \otimes d(q) \wedge d\left(q^{(2)}\right)+p^{(3)} q \otimes d(q) \wedge d\left(q^{(3)}\right)+p^{(3)} q^{(2)} \otimes d(q) \wedge d\left(q^{(4)}\right)+\ldots \\
& c_{1,-5}=p \otimes d\left(q^{(2)}\right) \wedge d\left(p q^{(4)}\right)+p \otimes d\left(p q^{(2)}\right) \wedge d\left(q^{(4)}\right)+\ldots \\
& c_{0,-4}=p \otimes d\left(p q^{(2)}\right) \wedge d\left(p q^{(3)}\right)+p \otimes d\left(q^{(3)}\right) \wedge d\left(p^{(2)} q^{(2)}\right)+\ldots \\
& c_{-1,-5}=p \otimes d\left(p^{(2)}\right) \wedge d\left(p q^{(6)}\right)+p \otimes d\left(p^{(3)}\right) \wedge d\left(q^{(6)}\right)+\ldots \\
& c_{-2,-6}=p \otimes d\left(p q^{(4)}\right) \wedge d\left(p^{(3)} q^{(3)}\right)+q \otimes d\left(p q^{(4)}\right) \wedge d\left(p^{(2)} q^{(4)}\right)+\ldots \\
& c_{-2,-4}=p \otimes d\left(p q^{(2)}\right) \wedge d\left(p^{(3)} q^{(3)}\right)+p \otimes d\left(p^{(3)} q\right) \wedge d\left(p q^{(4)}\right)+\ldots \\
& c_{-1,-3}=p \otimes d\left(q^{(2)}\right) \wedge d\left(p^{(3)} q^{(2)}\right)+p \otimes d\left(p^{(2)} q\right) \wedge d\left(p q^{(3)}\right)+\ldots \\
& c_{0,-2}=p \otimes d(p q) \wedge d\left(p q^{(2)}\right)+p \otimes d\left(q^{(2)}\right) \wedge d\left(p^{(2)} q\right)+\ldots \\
& c_{2,0}=p^{(2)} \otimes d(p) \wedge d(q)+p q^{(2)} \otimes d(q) \wedge d\left(q^{(2)}\right)+\ldots \\
& c_{-2,-2}=p \otimes d\left(p q^{(2)}\right) \wedge d\left(p^{(3)} q\right)+q \otimes d(q) \wedge d\left(p^{(3)} q^{(3)}\right)+\ldots \\
& c_{-2,0}=p \otimes d\left(p^{(2)}\right) \wedge d\left(p^{(2)} q\right)+p \otimes d(p q) \wedge d\left(p^{(3)}\right)+\ldots \\
& c_{-4,-2}=p \otimes d\left(p^{(3)}\right) \wedge d\left(p^{(3)} q^{(3)}\right)+q \otimes d\left(p^{(3)}\right) \wedge d\left(p^{(2)} q^{(4)}\right)+\ldots \\
& c_{-4,0}=p \otimes d\left(p^{(3)}\right) \wedge d\left(p^{(3)} q\right)+q \otimes d\left(p^{(3)}\right) \wedge d\left(p^{(2)} q^{(2)}\right)+\ldots \\
& c_{0,4}=\left(q^{(4)} \otimes(d(p) \wedge d(q))+\left(p^{(2)} q^{(3)}\right) \otimes d(p) \wedge d\left(p^{(2)}\right)+\ldots\right. \\
& c_{0,6}=q^{(6)} \otimes d(p) \wedge d(q)+p^{(2)} q^{(5)} \otimes d(p) \wedge d\left(p^{(2)}\right)+\ldots \\
& c_{-2,8}=q^{(7)} \otimes d(p) \wedge d\left(p^{(2)}\right)+p q^{(7)} \otimes d(p) \wedge d\left(p^{(3)}\right)+p^{(2)} q^{(7)} \otimes d\left(p^{(2)}\right) \wedge d\left(p^{(3)}\right) .
\end{aligned}
$$

6.4.2. Conjecture. The Lie algebra $\operatorname{Kap}_{4, B}(2 m)$ is not isomorphic to $\mathfrak{p o}_{\Pi}\left(2 m ; \underline{N}_{s}\right)$, and $\operatorname{Kap}_{2}(2 m)$ is not isomorphic to $\mathfrak{h}_{\Pi}(2 m ; \underline{N})$.

We verified this for small $m$. For $m=1, \operatorname{Kap}_{4, B}(2)$ is isomorphic to $\mathfrak{o}^{\prime}(3) \oplus \mathfrak{c}$, where $\mathfrak{c}$ is the 1-dimensional trivial center and is hence not isomorphic to $\mathfrak{p o}_{\Pi}\left(2 ; \underline{N}_{s}\right)$, which is solvable. For $m=2$, computer-aided computations show that the infinitesimal deformation corresponding to (68) is a nontrivial cocycle. To prove the conjecture, we must show that the cocycle is also not semitrivial. Of course, what we really need to know is what $\operatorname{Kap}_{4, B}(2 m)$ and its subalgebras $\operatorname{Kap}_{4, a}(2 m)$ are isomorphic to. We present some plausible conjectures.

6.4.3. Conjectures. 1. The Lie algebra Kap ${ }_{4,1}(2 \mathrm{~m})$ is a deform of the subalgebra in the Poisson algebra $\mathfrak{p o}\left(2 m ; \underline{N}_{s}\right)$ generated by functions $f \in \mathcal{O}\left(2 m ; \underline{N}_{s}\right)$ satisfying $\sum_{1 \leq i \leq 3} \frac{\partial^{2} f}{\partial p_{i} \partial q_{i}}=0$. (The quotient of this subalgebra modulo center is isomorphic to $\mathfrak{s l h}(2 m)$; see [LeP].)

2. The Lie algebra Kap $\operatorname{Kap}_{4,1}(2 m)$ is a deform of $\mathfrak{o}_{I}^{\prime}\left(2 m+1 ; \underline{N}_{s}\right)$ while $K_{a p} p_{4,0}(2 m)$ is a deform of a subalgebra in $\mathfrak{o}_{I}^{\prime}\left(2 m ; \underline{N}_{s}\right)$ (see $[\mathrm{LeP}]$ ).

The dimension of $H^{2}(\mathfrak{g} ; \mathfrak{g})$ is big and grows quickly with $m$. How can we select the needed deform? The Poisson algebra and its subalgebra consisting of harmonic functions have a center generated by constants, while $\operatorname{Kap}_{4,1}(2 m)$ is simple. Therefore, in the huge space of cocycles representing infinitesimal deformations, we need only select cocycles of the form

$$
f \otimes d(1) \wedge d(g)+\ldots
$$


and compare the global deforms corresponding to such cocycles with $\operatorname{Kap}_{4,1}(2 m)$. For small $m, \operatorname{dim} H^{2}(\mathfrak{g} ; \mathfrak{g})$ does not explode yet. For $m=2$ and $m=3$, we have $\operatorname{dim} H^{2}(\mathfrak{g} ; \mathfrak{g})=34$; all cocycles are integrable and all global deforms corresponding to them (if a representative is chosen carefully by means of coboundaries) are linear in the deformation parameter. For $m=2$ and $m=3$, there is only one cocycle of the form (103) (up to coboundaries). These cocycles are of degree 2. In degree 2 , there is only one cocycle for $m=3$, and there are five cocycles for $m=2$. Further investigations show that Conjecture 1 only holds for $m=2$; for $m=3$, the two algebras to be compared have different numbers of central extensions.

6.5. How to establish nonisomorphicy?. Skryabin [Sk] classified the filtered deforms of Hamiltonian Lie algebras $\mathfrak{h}_{\Pi}(2 m ; \underline{N})$. It remains to select which of them is the simple Lie algebra $\operatorname{Kap}_{4, B}(2 m) / \mathfrak{c} \simeq \operatorname{Kap}_{2}(2 m)$. We have not yet performed such an identification.

To find out if two given Lie algebras of the same dimension are isomorphic, Eick considered the following invariants in Ei] $15 \operatorname{dim} H^{1}(\mathfrak{g} ; \mathfrak{g})$ or rather $\operatorname{dim} \mathfrak{d} \mathfrak{e r}(\mathfrak{g})$, the order of the group $\operatorname{Aut}(\mathfrak{g})$, the number of elements in $\operatorname{Ann}(\mathfrak{g})$, and the order of $\operatorname{Exp}(\mathfrak{g})$.

Speaking of deforms, we can consider the action of $\operatorname{Aut}(\mathfrak{g})$ on the space of infinitesimal deformations, as in [KCh, $\mathrm{Ch}$.

For algebras of small dimension, there is still another approach, at least theoretically. We can compare identities that the algebras satisfy. A. A. Kirillov formulated the following analog of the Amitsur-Levitzki theorem, whose proof was only preprinted in the Keldysh Institute of Applied Mathematics in the 1980s (see [KOU] for a translation of one such preprint; the other preprints with related results by Kirillov, Kontsevich, and Molev have not yet been translated, but they were at least reviewed by Molev).

6.5.1. Theorem ([Ki $]$ ). Let $\mathfrak{g}$ be a simple Lie algebra of vector fields over a field of characteristic 0 . Let

$$
a_{k}\left(X_{1}, \ldots, X_{k}\right)=\sum_{\sigma \in S_{k}}(-1)^{\operatorname{sign} \sigma} \operatorname{ad}_{X_{\sigma(1)}} \ldots \operatorname{ad}_{X_{\sigma(k)}} .
$$

The identity $a_{k}\left(X_{1}, \ldots, X_{k}\right) \equiv 0$ for any $X_{1}, \ldots, X_{k} \in \mathfrak{g}$ holds

a. for $k \geq(n+1)^{2}$ if $\mathfrak{g}=\mathfrak{v e c t}(n)$,

b. for $k \geq n(2 n+5)$ if $\mathfrak{g}=\mathfrak{h}(2 n)$, and

c. for $k \geq 2 n^{2}+5 n+5$ if $\mathfrak{g}=\mathfrak{k}(2 n+1)$.

Dzhumadildaev suggested an interesting modification of emphasis in this train of thought, finding a hidden supersymmetry for an analog of antisymmetrizors with just $x$ instead of $\operatorname{ad}_{x}$ in (104). He also showed a relation to strongly homotopy algebras (for further details, see [Dzhu] and [LL]).

\section{ACKNOWLEDGEMENTS}

We are thankful to P. Grozman for his wonderful package SuperLie (see [Gr]). Special thanks are due to the referee for the helpful comments and to P. Zusmanovich, who informed us about important papers [Ei] and [SkT1]. S.B. was supported in part by the grant AD 065 NYUAD.

\footnotetext{
${ }^{15} \mathrm{An}$ almost exact quotation from [Ei]: "We say that a derivation $d \in \mathfrak{d e r}(\mathfrak{g})$ is p-nilpotent if $d^{p}=0$ holds. For a $p$-nilpotent derivation $d$, we define its exponential matrix $\exp d:=\sum_{0 \leq i \leq p-1} \frac{d^{i}}{i !}$. We call a $p$ nilpotent derivation $d$ an annihilator if $d^{i}(X) d^{j}(Y)=0$ for all $X, Y \in \mathfrak{g}$ and $i, j \geq 0$ with $i+j \geq p$. Let $\operatorname{Ann}(\mathfrak{g}) \subset \mathfrak{d} \mathfrak{r}(\mathfrak{g})$ denote the subset of annihilators. We define $\operatorname{Exp}(\mathfrak{g})$ to be the subgroup of Aut $(\mathfrak{g})$ generated by $\{\exp (d) \mid d \in \operatorname{Ann}(\mathfrak{g})\}$. We note that the order of every element $\exp (d)$ is equal to either $p$ or 1 . Hence, $\operatorname{Exp}(\mathfrak{g})$ is a subgroup of $\operatorname{Aut}(\mathfrak{g})$ generated by automorphisms of order $p . "$
} 


\section{REFERENCES}

[Al] A.A. Albert, Symmetric and alternate matrices in an arbitrary field, I, Trans. Amer. Math. Soc., 43:3 (1938), 386-436.

[BGP] G. Benkart, Th. Gregory, and A. Premet, The recognition theorem for graded Lie algebras in prime characteristic. American Mathematical Society, 2009, 145 pp.

[BGL1] S. Bouarroudj, P. Grozman, and D. Leites, Classification of simple finite dimensional modular Lie superalgebras with Cartan matrix, Symmetry, Integrability and Geometry: Methods and Applications (SIGMA), 5 (2009), 060, 63 pp; arXiv:math.RT/0710.5149.

[BGL2] S. Bouarroudj, P. Grozman, and D. Leites, Deforms of symmetric simple modular Lie superalgebras, arXiv: 0807.3054.

[BGL3] S. Bouarroudj, P. Grozman, and D. Leites, New simple modular Lie superalgebras as generalized Cartan prolongations, Functional Analysis and Its Applications, 42 (2008), no. 3, 161-168; arXiv:math.RT/0704.0130.

[BGLL] S. Bouarroudj, P. Grozman, A. Lebedev, and D. Leites, Divided power (co)homology. Presentations of simple finite dimensional modular Lie superalgebras with Cartan matrix, Homology, Homotopy and Applications, 12 (2010), no. 1, 237-278; arXiv:0911.0243.

[BGLLS] S. Bouarroudj, P. Grozman, A. Lebedev, D. Leites, and I. Shchepochkina, Simple prolongs of nonpositive parts of graded Lie algebras with Cartan matrix in characteristic 2, arXiv:1307.1551.

[BGLLS1] S. Bouarroudj, P. Grozman, A. Lebedev, D. Leites, and I. Shchepochkina, Simple vectorial Lie algebras in characteristic 2 , in preparation.

[BLLS] S. Bouarroudj, A. Lebedev, D. Leites, and I. Shchepochkina, Restricted simple Lie (super)algebras, classification of simple Lie superalgebras in characteristic 2, arXiv:1407.1695.

[BLW] S. Bouarroudj, A. Lebedev, and F. Wagemann, Deformations of the Lie algebra $\mathfrak{o}(5)$ in characteristics 3 and 2, Mathem. Notes, 89:6 (2011) 777-791; arXiv:0909.3572.

[Bro] G. Brown, Families of simple Lie algebras of characteristic two, Comm. Algebra, 23(3) (1995) 941954.

[Ch] N.G. Chebochko, Deformations of classical Lie algebras with a homogeneous root system in characteristic two. I, Sb. Math., 196 (2005) no. 9-10, 1371-1402.

[Del] P. Deligne, P. Etingof, D. Freed, L. Jeffrey, D. Kazhdan, J. Morgan, D. Morrison, and E. Witten, (eds.). Quantum fields and strings: a course for mathematicians. Material from the Special Year on Quantum Field Theory held at the Institute for Advanced Study, Princeton, NJ, $1996--97$. American Mathematical Society, Providence, RI; Institute for Advanced Study (IAS), Princeton, NJ, 1999. Vol. 1: xxii+723 pp.

[Dye] R.H. Dye, On the Arf invariant, J. Algebra, 53:1 (1978) 36-39.

[Dzh] A. Dzhumadildaev, Deformations of Lie algebra $W_{n}(\boldsymbol{m})$, Mathematics of the USSR-Sbornik, 66:1 (1990) $169-187$.

[Dzhu] A. Dzhumadildaev, 2p-commutator on differential operators of order $p$. Lett. Mathem. Physics, 104:7 (2014), 849-869.

[DzhK] A. Dzhumadildaev, and A.I. Kostrikin, Deformations of Lie algebra $W_{1}(m)$, Proceedings of the Steklov Institute of Mathematics, 148 (1980) 143-158.

[Ei] B. Eick, Some new simple Lie algebras in characteristic 2. J. Symb. Comput. 45 (2010) no.9, 943951.

[FG] D.E. Frohardt, and R.L.Griess, Automorphisms of modular Lie algebras, Nova J. Algebra Geom., 1 (1992) 339-345.

[Fu] D. Fuks (Fuchs), Cohomology of infinite dimensional Lie algebras, Consultants Bureau, NY, 1986.

[GJu] A. Grishkov, On simple Lie algebras over a field of characteristic 2, J. Algebra, 363 (2012) 14-18.

[GZ] A. Grishkov, P. Zusmanovich, Deformations of current Lie algebras. I. Small algebras in characteristic 2; arXiv: 1410.3645

[Gr] P. Grozman, SuperLie, http://www.equaonline.com/math/SuperLie

[GL] P. Grozman, and D. Leites, Structures of $G(2)$ type and nonintegrable distributions in characteristic p, Lett. Math. Phys. 74 (2005) no. 3, 229-262; arXiv:math.RT/0509400

[Ju] G. Jurman, A family of simple Lie algebras in characteristic two, J. Algebra, 271 (2004) no. 2, $454-481$.

[Kap2] I. Kaplansky, Some simple Lie algebras in characteristic 2, In: Lie algebras and related topics (New Brunswick, N.J., 1981). Lecture Notes in Math., 933, Springer, Berlin, (1982), 127-129. 
[Ki] A. Kirillov, On identities in the Lie algebras of vector fields Vestnik Mosk. universiteta. Ser. 1 Matematika i mehanika. (1989) no.2, 11-17. (Russian)

[KOU] A. Kirillov, V. Ovsienko, and O. Udalova, Identities in the Lie algebras of vector fields on the real line, Selecta Mathematica Sovietica, 10 (1991) no.1, 7-17.

$[\mathrm{KT}] \mathrm{S}$. Konstein, and I. Tyutin, The deformations of antibracket with even and odd deformation parameters, arXiv:1011.5807.

[KD] A.I. Kostrikin, and A.S. Dzhumadildaev, Modular Lie algebras: new trends. In: Yu. Bahturin (ed.), Algebra. Proc. of the International Algebraic Conference on the Occasion of the 90th Birthday of A.G. Kurosh. de Gruyter, 2000, 181-203.

[KCh] M.I. Kuznetsov, and N.G. Chebochko, Deformations of classical Lie algebras, Sb. Math., 191 (2000) no. $7-8,1171-1190$.

[KuJa] M.I. Kuznetsov, and V.A. Jakovlev, On exceptional simple Lie algebras of series R, In: 3rd Intn. conference on algebra, Krasnoyarsk, 1993, Theses of reports, 411.

[LeP] A. Lebedev, Analogs of the orthogonal, Hamiltonian, Poisson, and contact Lie superalgebras in characteristic 2, J. Nonlinear Math. Phys., 17, Special issue in memory of F. Berezin, (2010) 217251.

[LL] A. Lebedev, and D. Leites, Hidden supersymmetry of commutators: the Amitsur-Levitzki identity and the Dzhumadildaev brackets, in preparation.

[Ltow] D. Leites, Towards classification of simple finite dimensional modular Lie superalgebras, J. Prime Res. Math., 3 (2007) 101-110; arXiv:0710.5638.

[Lsos] D. Leites (ed.) Seminar on supersymmetry (v. 1. Algebra and Calculus: Main chapters), (J. Bernstein, D. Leites, V. Molotkov, and V. Shander), MCCME, Moscow, 410 pp (in Russian; a version in English is in preparation but available for perusal).

[LSh1] D. Leites, and I. Shchepochkina, Classification of the simple Lie superalgebras of vector fields, Preprint MPIM-2003-28 (Available at http://www.mpi-bonn.mpg.de)

[LSh2] D. Leites, and I. Shchepochkina, How to quantize the antibracket, Theor. and Math. Physics, 126 (2001) no. 3, 339-369; arXiv:math-ph/0510048.

[LLg] Dong Liu, and Lei Lin, On the variations of $G_{2}$, Chin. Ann. Math., 24B (2003) no.3, 387-294.

[Pre] A. Premet, Algebraic groups associated with Cartan Lie p-algebras, Mathematics of the USSRSbornik, 50:1 (1985) 85-97.

[Shch] I. Shchepochkina, How to realize Lie algebras by vector fields, Theor. Mat. Fiz., 147 (2006) no. 3, 821-838; arXiv:math.RT/0509472

[Sh] G.Y. Shen, Variations of the classical Lie algebra G_2 in low characteristics, Nova J. Algebra Geom., 2 (1993) no.3, 217-243.

[Sk] S.M. Skryabin, Classification of Hamiltonian forms over divided power algebras, Math. of the USSRSbornik, 69:1 (1991) 121-141

[SkT1] S.M. Skryabin, Toral rank one simple Lie algebras of low characteristics. J. of Algebra, 200 (1998), no. 2, 650-700

[S] H. Strade, Simple Lie algebras over fields of positive characteristic. I-III, de Gruyter Expositions in Mathematics, Walter de Gruyter \& Co., Berlin, (2004) viii+540 pp; (2009) vi+385pp; (2012) x+239pp.

[Vi1] F. Viviani, Simple finite group schemes and their infinitesimal deformations, Rend. Sem. Mat. Univ. Politec. Torino, 68 (2010) 171-182; arXiv:0811.2668.

[WK] B.Ju. Weisfeiler, and V.G. Kac, Exponentials in Lie algebras of characteristic p, Mathematics of the USSR-Izvestiya, 5:4 (1971) 777-803.

${ }^{1}$ New York University Abu Dhabi, Division of Science and Mathematics, P.O. Box 129188, United Arab Emirates; sofiane.Bouarroudj@nyu.edu, ${ }^{2}$ Equa Simulation AB, Stockholm, Sweden; aleXeylalexeyl@mail.ru, ${ }^{3}$ Department of Mathematics, Stockholm University, Roslagsv. 101, Kräftriket hus 6, SE-106 91 Stockholm, Sweden; mleites@Math.su.se, ${ }^{4}$ Independent UniVersity of Moscow, Bolshoj Vlasievsky Per, dom 11, RU-119 002 Moscow, Russia;iRina@mCCME.RU 